\title{
Computational Modelling of Multiscale, Multiphase Fluid Mixtures with Application to Tumour Growth.
}

\author{
J. Collis ${ }^{\text {a }}$ M. E. Hubbard ${ }^{\text {a }}$, R. D. O'Dea ${ }^{\mathrm{a}}$ \\ ${ }^{a}$ School of Mathematical Sciences, University of Nottingham, University Park, Nottingham \\ $N G 7$ 2RD, UK
}

\begin{abstract}
In this work we consider the discretization of a recently formulated [18] multiscale model for drug- and nutrient-limited tumour growth. The key contribution of this work is the proposal of a discontinuous Galerkin finite element scheme which incorporates a non-standard coupling across a singular surface, and the presentation of full details of a suitable discretization for the coupled flow and transport systems, such as that arising in [18] and other similar works. We demonstrate the application of the proposed discretizations via representative numerical experiments; furthermore, we present a short numerical study of convergence for the proposed microscale scheme, in which we observe optimal rates of convergence for sufficiently smooth data.
\end{abstract}

Keywords: Numerical simulations, finite elements, porous media, cancer modelling

\section{Introduction}

Over many years, mathematicians have sought effective means of incorporating effects occurring on multiple spatial and temporal scales into mechanistic models, whether this is in the classical settings of homogenization via asymptotic expansions and volume averaging [19], or the more contemporary setting

Email addresses: Joe.Collis@nottingham.ac.uk (J. Collis), Matthew.Hubbard@nottingham.ac.uk (M. E. Hubbard), Reuben.0'Dea@nottingham.ac.uk (R. D. O'Dea)

Preprint submitted to Elsevier

July 19, 2016 
of multiscale numerical methods, see e.g. [12, 22, 23]. Multiscale effects are of interest to many communities of applied mathematicians, such as those studying groundwater flow $[28,39]$ or biological systems [31, 37, 41]. As these techniques become better developed, we observe an increased level of their application across an ever greater range of physical and biological systems, inevitably driving further development of both analytical and computational techniques.

A specific application that has garnered much interest is the study of cancer as a multiscale system. There have been extensive developments in the mathematical and computational modelling of tumour growth since the middle portion of the last century [9]. However, in the last few decades there has been an increased focus on incorporating mechanisms occurring on multiple scales in an effective manner, see e.g. [1-4, 25, 29, 33-36, 43] and the references therein. Understanding the dependence of a tumour's evolving microstructure and microvasculature is vital for making predictions regarding the potential efficacy of a drug in the treatment of a tumour [34, 35].

In the companion article to this work [18], we consider an asymptotic analysis of a multiphase fluid dynamics model for avascular tumour growth of the type exploited in $[11,27]$. This analysis allows us to derive a system of equations that describes tumour growth at a lengthscale associated with the full extent of the tumour tissue, that is explicitly dependent on a microscale formulation describing the microstructural properties of the tumour. The resulting microscale problem comprises a system of coupled tensor Stokes problems, the macroscale problem, a coupled system of nonlinear hyperbolic transport equations, further coupled to an elliptic flow equation. The analysis of [18] represents an extension of the multiscale analyses of $[31,42,43]$, as we consider growth that is dependent on the tissue composition in a multiscale framework as a means of incorporating interstitial growth. However, the relative complexity of the microand macroscale systems obtained in [18], compared to those in [31, 42, 43], necessitates increased sophistication in their discretization.

In this article, we address the challenges associated with defining suitable discretizations for both systems of equations. For the microscale system, we 
consider a means of incorporating the non-standard coupling of flow across a quasi-stationary internal surface into a discontinuous Galerkin (dG) finite element (FE) discretization which, to the authors' knowledge, has not been considered elsewhere in the literature (cf. [10] and references cited therein). For the macroscale system, the primary difficulty arises in obtaining a suitably stable discretization for the complex coupled system of nonlinear partial differential equations (PDEs), that does not suffer from spurious numerical oscillations. As such, we present a full description of the dG FE and mixed Raviart-Thomas (RT)/dG FE discretizations employed in [18]. Additionally, we present a short numerical study of the convergence of the method proposed for the microscale

problem as well as a selection of representative numerical experiments for the macroscale problem.

This article is organized as follows. In section 2 we recall the microscale and macroscale models presented in [18] governing tumour growth together with passive transport of drug and nutrient, and in section 3 we introduce the discretization employed in their solution. In section 4 we present a short numerical study of the convergence of the discretization of microscale model and a selection of numerical experiments demonstrating the dynamics of the macroscale system. Finally, in section 5 we make some concluding remarks and highlight ongoing and future work.

\section{Model Description}

In this section we provide a brief summary of the underlying conceptual model describing drug- and nutrient-regulated growth and response of a tumour, and transport of passive solutes as described in [18], as well as brief details of the formulation arising from a multiple scales analysis contained therein, the solution of which forms the principal focus of the current work.

\subsection{Conceptual Model}

We consider a multiphase fluid dynamics model of avascular tumour growth and transport of passive solutes based on that employed in [11, 27], in which 
detailed justification for the application of models of this type is provided. We consider a formulation that is characterized by two lengthscales that are strongly separated, referred to as the microscale and the macroscale. Under this assumption, described formally below, and strong interphase drag so that all phases move with a common velocity (motivated by such works as [24, 32]), we are able to derive an effective description of growth and transport on the macroscale (corresponding to the lengthscale of the tumour tissue), explicitly incorporating microscale tumour dynamics.

We consider a region of tumour tissue, $\Omega_{L} \subset \mathbb{R}^{d}(d=2,3)$, as an idealized porous medium that consists of a multicomponent mixture, comprising $N_{\theta}$ interacting phases, saturated with a viscous Newtonian fluid, and subsequently referred to as the mixture and interstitial fluid, respectively. We assume that within $\Omega_{L}$ there is a spatially periodic microstructure, which we denote $\Omega_{\ell}$, that further defines two subdomains $\Omega_{1}$ and $\Omega_{2}$, corresponding to the regions that contain the multicomponent mixture and the interstitial fluid, respectively. We further assume that the free interface between $\Omega_{1}$ and $\Omega_{2}$, denoted $\Gamma$, is sharp, and that phase transition may occur on this singular surface; where the movement of $\Gamma$ is determined by tissue growth. A schematic diagram of this geometry is shown in Figure 1. Additionally, we assume that the porous medium may be characterized by two distinct lengthscales $(\ell$, corresponding to the lengthscale associated with the periodic microstructure and $L$, corresponding to the lengthscale of the tissue) that are well-separated. To this end, we introduce a small, dimensionless parameter $0<\epsilon \ll 1$ defined by

$$
\epsilon=\frac{\ell}{L}
$$

Under the assumption of strong interphase viscous drag, so that all phases in the multicomponent mixture move with a common velocity, we write all dependent variables $\Upsilon$ as a multiple scales expansion in $\epsilon$ of the type

$$
\Upsilon(\cdot ; \epsilon)=\sum_{i=0}^{\infty} \epsilon^{i} \Upsilon^{(i)}(\cdot)
$$

as a means to analyse the leading order, i.e. $\mathcal{O}(1)$, behaviour of the system. As a 
result of the linearity of the system, to leading order, we are able to decouple the micro- and macroscale flow description through a Darcy ansatz on the leading order flow, $\boldsymbol{u}^{(0)}$, and first order pressure, $p^{(1)}$, of the form

$$
\boldsymbol{u}^{(0)}=-\boldsymbol{K} \nabla p^{(0)} \quad \text { and } \quad p^{(1)}=-\boldsymbol{a} \cdot \nabla p^{(0)}+\bar{p}
$$

where $p^{(0)}$ denotes the microscale invariant first order pressure, $\bar{p}$ denotes the mean value of $p^{(1)}$ in $\Omega$, and the permeability tensor $\boldsymbol{K}$ and vector $\boldsymbol{a}$ parameterize the microscale variation of $\boldsymbol{u}^{(0)}$ and $p^{(1)}$, respectively. By substituting this ansatz into the systems of equations obtained at leading and first order, i.e. $\mathcal{O}(1)$ and $\mathcal{O}(\epsilon)$, we are able to obtain a pair tensor Stokes problem for $\boldsymbol{K}$ and $\boldsymbol{a}$ in $\Omega$, coupled via suitable conditions on the interface $\Gamma$. This system is described formally in section 2.2. We note that these equations are stationary and independent of any macroscale variation. Thus, all we require to parameterize the unsteady macroscale problem across the full extent of the tissue is the solution of a stationary problem on a single periodic unit. Then, via spatial averaging, we derive an effective system of equations on the macroscale comprising a coupled system of nonlinear advection-reaction PDEs governing the evolution of the concentration of $\operatorname{drug} c$, concentration of nutrient $n$, and volume fractions of the components of the mixture $\boldsymbol{\theta}=\left(\theta_{1}, \theta_{2}, \ldots, \theta_{N_{\theta}}\right)^{\top}$, and an elliptic PDE in mixed form governing the flow, described by the spatially averaged, leading order velocity $\boldsymbol{u}$ and leading order pressure $p$. We describe this system formally in section 2.3.

\subsection{Microscale PDE Model}

In addition to the notation introduced in section 2.1 , we further denote the inward unit normal to $\Omega_{1}$ on $\Gamma$ by $\boldsymbol{n}_{\Gamma}$, the unit tangent(s) to $\Gamma$ by $\boldsymbol{\tau}_{\Gamma}$, and the external boundary of $\Omega_{\ell}$ by $\partial \Omega_{\ell}$. A schematic diagram of the microscale geometry, demonstrating the notation introduced here, is shown in Figure 1. We let $\mu_{1}$ and $\mu_{2}$ denote the kinematic viscosity of the fluids in $\Omega_{1}$ and $\Omega_{2}$, 
respectively, and further define

$$
\mu:= \begin{cases}\mu_{1} & \text { in } \Omega_{1}, \\ \mu_{2} & \text { in } \Omega_{2} .\end{cases}
$$

In addition to a system of PDEs on $\Omega_{\ell}$, we define a set of interface conditions on $\Gamma$. To this end, we first define the \pm sides of the interface $\Gamma$, i.e. for a scalar quantity $a$ defined in both $\Omega_{1}$ and $\Omega_{2}$, we define the quantities $a^{ \pm}$by

$$
a^{ \pm}:=\lim _{\varepsilon \rightarrow 0^{+}} a\left(\boldsymbol{x} \mp \varepsilon \boldsymbol{n}_{\Gamma}, t\right) \quad \forall \boldsymbol{x} \in \Gamma,
$$

where $\boldsymbol{x}$ denotes the microscale spatial variable, which we extend componentwise for vector or tensor quantities. We futher introduce the notation

$$
[a]_{-}^{+}:=a^{-}-a^{+}
$$

for a scalar quantity $a$, which we extend to vector or tensor quantities componentwise. Finally, we denote the gradient, Laplacian and rate of strain operators corresponding to differentiation with respect to the microscale spatial variable by $\nabla_{\boldsymbol{x}}, \Delta_{\boldsymbol{x}}$, and $\boldsymbol{D}_{\boldsymbol{x}}$, respectively. The leading order microscale dependence of

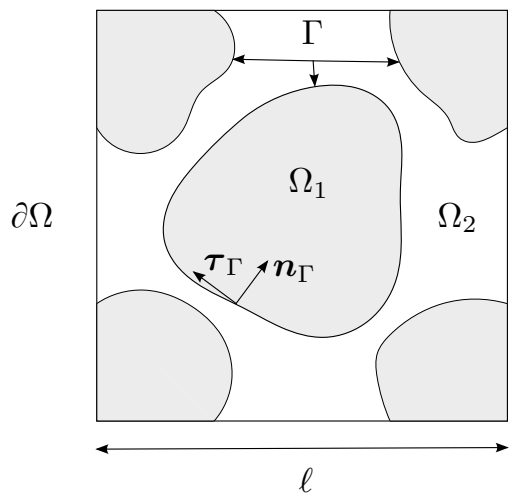

Figure 1: Schematic of a single unit $\Omega_{l}$ of the periodic microscale problem. Diagram adapted from [31, Fig. 1].

the system is now given by a vector $\boldsymbol{a}$ and a permeability tensor $\boldsymbol{K}$ defined on $\Omega_{\ell}$ that satisfy

$$
\left.\begin{array}{rlrl}
-\nabla_{\boldsymbol{x}} \boldsymbol{a}+\mu \Delta_{\boldsymbol{x}} \boldsymbol{K}^{\top} & =-\boldsymbol{I} & & \forall \boldsymbol{x} \in \Omega_{\ell}, \\
\nabla_{\boldsymbol{x}} \cdot \boldsymbol{K}^{\top} & =\mathbf{0} & & \forall \boldsymbol{x} \in \Omega_{\ell},
\end{array}\right\}
$$


subject to the following conditions on the interface $\Gamma$

$$
\left.\begin{array}{rlr}
{\left[-\boldsymbol{a} \otimes \boldsymbol{n}_{\Gamma}+\mu \boldsymbol{D}_{\boldsymbol{x}}\left(\boldsymbol{K}^{\top}\right) \boldsymbol{n}_{\Gamma}\right]_{-}^{+}} & =\mathbf{0} & \forall \boldsymbol{x} \in \Gamma, \\
{\left[\boldsymbol{K}^{\top} \boldsymbol{\tau}_{\Gamma}\right]_{-}^{+}=\mathbf{0}} & \forall \boldsymbol{x} \in \Gamma, \\
\left(\boldsymbol{K}^{\top} \boldsymbol{n}_{\Gamma}\right)^{+}=\mathbf{0} & \forall \boldsymbol{x} \in \Gamma, \\
\left(\boldsymbol{K}^{\top} \boldsymbol{n}_{\Gamma}\right)^{-}=\mathbf{0} & \forall \boldsymbol{x} \in \Gamma,
\end{array}\right\}
$$

and periodic boundary conditions on $\partial \Omega_{\ell}$ for all components of $\boldsymbol{K}$ and all com-

ponents of $-\boldsymbol{a} \otimes \boldsymbol{n}_{\Gamma}+\mu \boldsymbol{D}_{\boldsymbol{x}}\left(\boldsymbol{K}^{\top}\right) \boldsymbol{n}_{\Gamma}$. The Stokes system (7) and interface conditions (8) are obtained by substituting the Darcy ansatz (3) into the flow equations and interface conditions obtained at leading and first order from our asymptotic expansion. The tensor Stokes equations for permeability are standard, see e.g. [39]. The interface conditions are a result of the imposition of continuity of stress and velocity across $\Gamma$, and the observation in [18] that, under a suitable choice of scaling, the interface is quasi-static to leading order.

We highlight that the combination of slip and no-penetration given for the interface condition is non-standard, and, as such, must be considered carefully when formulating the discretization of this problem in section 3.1.

We note that the system given by (7), (8), and periodicity is not sufficient to uniquely specify the vector $\boldsymbol{a}$. As such, we further specify that $\boldsymbol{a}$ is mean-free on $\Omega_{\ell}$, i.e.

$$
\int_{\Omega_{\ell}} \boldsymbol{a} d \boldsymbol{x}=\mathbf{0}
$$

\subsection{Macroscale PDE Model}

Prior to defining the macroscale model, we introduce additional notation for selected macroscale quantities. We define the following spatial averages for microscale-varying quantities over $\Omega_{1}, \Omega_{2}$, and $\Gamma$ by

$$
\langle g\rangle_{1}:=\frac{1}{\left|\Omega_{\ell}\right|} \int_{\Omega_{1}} g d \boldsymbol{x}, \quad\langle g\rangle_{2}:=\frac{1}{\left|\Omega_{\ell}\right|} \int_{\Omega_{2}} g d \boldsymbol{x}, \quad \text { and }\langle g\rangle_{\Gamma}:=\frac{1}{\left|\Omega_{\ell}\right|} \int_{\Gamma} g d s,
$$

respectively, and introduce the notation $\langle\cdot\rangle_{\Omega_{\ell}}:=\langle\cdot\rangle_{1}+\langle\cdot\rangle_{2}$. We define the porosity of the material, $\phi$, by

$$
\phi:=\frac{\left|\Omega_{1}\right|}{\left|\Omega_{\ell}\right|} .
$$


We further denote the exterior boundary of $\Omega_{L}$ by $\partial \Omega_{L}$, and let $\boldsymbol{n}$ denote the outward unit normal to $\partial \Omega_{L}$. We define $\boldsymbol{X}$ to be the dimensionless macroscale spatial variable related to $\boldsymbol{x}$ via $\epsilon \boldsymbol{X}=\boldsymbol{x}$, and denote the gradient and divergence operators corresponding to differentiation with respect to the macroscale variable by $\nabla_{\boldsymbol{X}}$ and $\nabla_{\boldsymbol{X}}$, respectively. Then, for a given vector field $\boldsymbol{b}$, we define the inflow portion of the boundary $\partial \Omega_{L}$ by

$$
\Lambda^{-}(\boldsymbol{b}):=\left\{\boldsymbol{X} \in \partial \Omega_{L}: \boldsymbol{b}(\boldsymbol{X}) \cdot \boldsymbol{n}<0\right\} .
$$

We proceed now by setting out this system of PDEs. The flow problem is given in mixed form by

$$
\left.\begin{array}{rlrl}
\boldsymbol{u}+\langle\boldsymbol{K}\rangle_{\Omega} \nabla_{\boldsymbol{X}} p & =0 & & \forall \boldsymbol{X} \in \Omega_{L}, \\
\nabla_{\boldsymbol{X}} \cdot \boldsymbol{u} & =f(\boldsymbol{X} ; \boldsymbol{\theta}) & & \forall \boldsymbol{X} \in \Omega_{L},
\end{array}\right\}
$$

subject to the boundary condition

$$
p=p_{D} \quad \forall \boldsymbol{X} \in \partial \Omega_{L} .
$$

Note that we adopt the notation $(\cdot ; \cdot)$ to emphasize the potentially nonlinear dependence of coefficients in the PDE system on dependent variables whose evolution is governed by other equations in the system; for example, the source term in (13), $f(\boldsymbol{X} ; \boldsymbol{\theta})$, is spatially varying and has a functional dependence on $\theta$.

In the following, we present the governing transport equations, together with associated boundary and initial conditions, using generic reaction, source, boundary, and initial data. The specific form of the reaction, source, and forcing terms are set out explicitly in appendix $\mathrm{A}$ as a particular choice of tumour growth model. The boundary and initial data employed in the numerical experiments in section 4.2 are prescribed therein. However, throughout this work we choose smooth boundary and initial data to reduce any analytical complexity regarding the well-posedness of the macroscale system. The equation governing the volume fractions of the components of the mixture is given by

$$
\frac{\partial \theta_{i}}{\partial t}+\tilde{\boldsymbol{u}} \cdot \nabla_{\boldsymbol{X}} \theta_{i}+\mathcal{R}_{\theta, i}(\boldsymbol{X} ; \boldsymbol{\theta}, c, n) \theta_{i}=g_{\theta, i}(\boldsymbol{X} ; \boldsymbol{\theta}, c, n) \quad \forall \boldsymbol{X} \in \Omega_{L},
$$


for $1 \leqslant i \leqslant N_{\theta}$, where $\mathcal{R}_{\theta, i}$ and $g_{\theta, i}$ denote reaction and source terms, respectively, and $\tilde{\boldsymbol{u}}$ denotes the average velocity of the mixture, defined by

$$
\tilde{\boldsymbol{u}}:=\langle\boldsymbol{K}\rangle_{1}\langle\boldsymbol{K}\rangle_{\Omega_{\ell}}^{-1} \boldsymbol{u}
$$

We further require that $\boldsymbol{\theta}$ is subject to the boundary condition

$$
\boldsymbol{\theta}(\boldsymbol{X}, t)=\boldsymbol{\Theta}(t) \quad \forall \boldsymbol{X} \in \Lambda^{-}(\tilde{\boldsymbol{u}}),
$$

and the initial condition

$$
\boldsymbol{\theta}(\boldsymbol{X}, 0)=\boldsymbol{\Theta}_{0}(\boldsymbol{X}) \quad \forall \boldsymbol{X} \in \Omega_{L} .
$$

The equation governing the evolution of the concentration of drug is given by

$$
\frac{\partial c}{\partial t}+\left(\boldsymbol{u} \cdot \nabla_{\boldsymbol{X}}\right) c+\mathcal{R}_{c}(\boldsymbol{X} ; \boldsymbol{\theta}) c=0 \quad \forall \boldsymbol{X} \in \Omega_{L},
$$

where $\mathcal{R}_{c}$ denotes a reaction term; subject to the boundary condition

$$
c(\boldsymbol{X}, t)=C(t) \quad \forall \boldsymbol{X} \in \Lambda^{-}(\boldsymbol{u}),
$$

and the initial condition

$$
c(\boldsymbol{X}, 0)=C_{0}(\boldsymbol{X}) \quad \forall \boldsymbol{X} \in \Omega_{L} .
$$

Finally, the equation governing the evolution of the concentration of nutrient is given by

$$
\frac{\partial n}{\partial t}+\left(\boldsymbol{u} \cdot \nabla_{\boldsymbol{X}}\right) n+\mathcal{R}_{n}(\boldsymbol{X} ; \boldsymbol{\theta}) n=0 \quad \forall \boldsymbol{X} \in \Omega_{L},
$$

where $\mathcal{R}_{n}$ denotes a reaction term; subject to the boundary condition

$$
n(\boldsymbol{X}, t)=N(t) \quad \forall \boldsymbol{X} \in \Lambda^{-}(\boldsymbol{u}),
$$

and the initial condition

$$
n(\boldsymbol{X}, 0)=N_{0}(\boldsymbol{X}) \quad \forall \boldsymbol{X} \in \Omega_{L} .
$$




\section{Discretization}

In this section we introduce discretizations of the microscale system introduced in section 2.2 and the macroscale system introduced in section 2.3. Prior to this, however, we briefly discuss our choice of numerical methods employed in sections 3.1 and 3.2 for the spatial discretization of the micro- and macroscale systems. Despite restricting our attention to FE methods, we are still presented with a range of suitable discretizations.

For the microscale problem, there are many spaces of conforming and nonconforming finite element methods that yield stable numerical methods, as discussed in e.g. [16]. In this work, we employ a dG FE method similar to that presented in [44]. While the comparative costs and benefits of dG FE methods are discussed extensively in the literature, see e.g. [17], for the numerical examples presented where standard conforming methods would require fewer degrees of freedom for equivalent accuracy. However, we highlight that $\mathrm{dG}$ methods are stable for a wider range of approximation spaces than standard conforming methods.

When considering a geometrically complex pore structure, such as those found in biological materials pertinent to applications of the model developed in [18], dG methods constructed on general polytopes/polyhedra provide a means of decoupling the dimensionality of the approximation space and geometrical complexity, see e.g. the review article [8]. Furthermore, the discontinuous nature of the approximation facilitates straightforward application of $h p$-refinement algorithms. So while for the simple geometries and methods considered in the current work it is not apparent that dG FE is the optimal method, it is employed as a precursor to wider application of dG FE methods on general polytopes/polyhedra in biomedical applications where there are benefits in terms of implementational ease and computational cost compared to standard conforming/non-conforming methods or conforming composite methods.

In the case of the macroscale flow equation, there are again a range of suitable FE methods available including $H^{1}$-conforming, dG, and $H$ (div)-conforming el- 
ements. As we consider the Darcy flow equation (13) in mixed form, it is natural for us to employ an $H$ (div)-conforming element [16] and, as such, we choose the Raviart-Thomas element [30, 38]. Physical motivations for selecting this class of conforming element are discussed briefly in section 3.2.1, as are alternative choices of $H(\mathrm{div})$-conforming elements. There is a more restrictive choice of (standard) FE methods for the transport equations for the macroscale model. We highlight here two families of methods, stabilised (continuous) methods and dG; here we adopt a dG FE method as described in [26]. Again, we refer to [17] for a discussion on the benefits of $\mathrm{dG}$ FE methods.

\subsection{Discretization of the Microscale System}

We consider conforming, shape-regular meshes $\mathcal{T}_{h}$ that partition $\Omega_{\ell}$ into disjoint open subdomains $\kappa$, such that $\overline{\Omega_{\ell}}=\bigcup_{\kappa \in \mathcal{T}_{h}} \bar{\kappa}$ and each element is the image of a reference element $\hat{\kappa}$ under the affine map $\mathcal{F}_{\kappa}: \hat{\kappa} \rightarrow \kappa$. We specify that $\mathcal{T}_{h}$ respects the decomposition of the domain $\Omega_{\ell}=\Omega_{1} \cup \Omega_{2}$ in the sense that a given element $\kappa$ is solely contained within $\Omega_{1}$ or $\Omega_{2}$.

Given $\kappa \in \mathcal{T}_{h}$, we denote by $\partial \kappa$ the boundary of $\kappa$, and by $\boldsymbol{n}_{\kappa}$ and $\boldsymbol{\tau}_{\kappa}$ the unit outward normal and tangent(s) to $\partial \kappa$, respectively, where $\tau_{\kappa}$ is oriented on each element boundary such that its definition is consistent for adjacent elements. We denote by $h_{\kappa}$ the element diameter, and introduce the mesh function $h:=\max \left\{h_{\kappa}: \kappa \in \mathcal{T}_{h}\right\}$. If the intersection $F=\bar{\kappa} \cap \bar{\kappa}^{\prime}$ between two elements $\kappa, \kappa^{\prime} \in \mathcal{T}_{h}$ is a proper line segment, then we refer to $F$ as an interior face. As we consider periodic boundary conditions, we impose the condition that for each face on the exterior boundary of $\mathcal{T}_{h}$, there is a corresponding face on the opposite side of the mesh. As such, we may identify these faces with each other and view the two elements that adjoin this face as being neighbours in the mesh. As a consequence, all faces in the mesh are interior faces. Similarly, if $F=\bar{\kappa} \cap \Gamma$ is a proper line segment, we refer to $F$ as an interfacial face. We denote the sets of all interior and interfacial faces as $\mathcal{E}_{I}\left(\mathcal{T}_{h}\right)$ and $\mathcal{E}_{\Gamma}\left(\mathcal{T}_{h}\right)$, respectively; and define the set of all non-interfacial faces by $\mathcal{E}\left(\mathcal{T}_{h}\right):=\mathcal{E}_{I}\left(\mathcal{T}_{h}\right) \backslash \mathcal{E}_{\Gamma}\left(\mathcal{T}_{h}\right)$. We further specify that faces (interior and interfacial) are linear. A schematic diagram of 


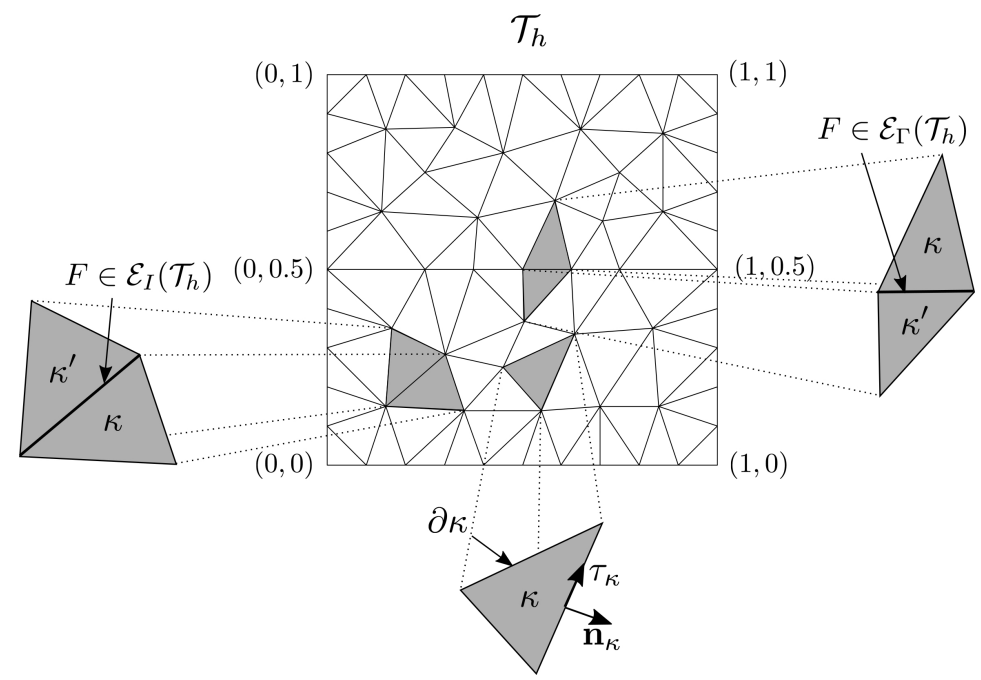

Figure 2: A schematic of the mesh, $\mathcal{T}_{h}$, discretizing the domain $\Omega_{\ell}=\Omega_{1} \cup \Omega_{2}$, where $\Omega_{1}=[0,1] \times[0,0.5]$ and $\Omega_{2}=[0,1] \times[0.5,1]$.

a sample mesh is shown in Figure 2.

We next define the trace operators used in the formulation of the finite element method. Let $F$ be an interior face shared by elements $\kappa_{1}$ and $\kappa_{2}$. For a scalar function $q$ that is smooth on each $\kappa \in \mathcal{T}_{h}$, we define the average and jump across $F$ by

$$
\{q\}:=\frac{1}{2}\left(\left.q\right|_{\kappa_{1}}+\left.q\right|_{\kappa_{2}}\right) \quad \text { and } \quad \llbracket q \rrbracket:=\left.q\right|_{\kappa_{1}} \boldsymbol{n}_{\kappa_{1}}+\left.q\right|_{\kappa_{2}} \boldsymbol{n}_{\kappa_{2}},
$$

where $\left.q\right|_{\kappa}$ denotes the restriction of the function $q$ to $\kappa$. If $\boldsymbol{v}$ is a piecewise smooth vector field, we define the average and jump of $\boldsymbol{v}$ on $F$ by

$$
\{\boldsymbol{v}\}:=\frac{1}{2}\left(\left.\boldsymbol{v}\right|_{\kappa_{1}}+\left.\boldsymbol{v}\right|_{\kappa_{2}}\right) \quad \text { and } \quad \llbracket \boldsymbol{v} \rrbracket:=\left.\boldsymbol{v}\right|_{\kappa_{1}} \cdot \boldsymbol{n}_{\kappa_{1}}+\left.\boldsymbol{v}\right|_{\kappa_{2}} \cdot \boldsymbol{n}_{\kappa_{2}},
$$

and the tensor jump of $\boldsymbol{v}$ on $F$ by

$$
\underline{\llbracket \boldsymbol{v} \rrbracket}:=\left.\boldsymbol{v}\right|_{\kappa_{1}} \otimes \boldsymbol{n}_{\kappa_{1}}+\left.\boldsymbol{v}\right|_{\kappa_{2}} \otimes \boldsymbol{n}_{\kappa_{2}} .
$$

If $\boldsymbol{T}$ is a piecewise smooth tensor field, we define the average and jump of $\boldsymbol{T}$ on $F$ by

$$
\{\boldsymbol{T}\}:=\frac{1}{2}\left(\left.\boldsymbol{T}\right|_{\kappa_{1}}+\left.\boldsymbol{T}\right|_{\kappa_{2}}\right) \quad \text { and } \quad \llbracket \boldsymbol{T} \rrbracket:=\left.\boldsymbol{T}\right|_{\kappa_{1}} \boldsymbol{n}_{\kappa_{1}}+\left.\boldsymbol{T}\right|_{\kappa_{2}} \boldsymbol{n}_{\kappa_{2}} .
$$


For $k \in \mathbb{N}_{0}$ and $\kappa \in \mathcal{T}_{h}$ we denote by $\mathcal{P}_{k}(\kappa)$ the space of polynomials of degree at most $k$ on $\kappa$. Under this notation, we define the dG FE spaces by

$$
S_{h, k}\left(\mathcal{T}_{h}\right):=\left\{v \in L^{2}\left(\Omega_{\ell}\right):\left.v\right|_{\kappa} \circ \mathcal{F}_{\kappa} \in \mathcal{P}_{k}(\hat{\kappa}), \kappa \in \mathcal{T}_{h}\right\}
$$

We now consider a dG FE method for the numerical approximation of systems of equations of the form given in (7) subject to the boundary conditions (8). To this end, we define the following finite element spaces

$$
\boldsymbol{V}:=\left(S_{h, k+1}\left(\mathcal{T}_{h}\right)\right)^{d} \quad \text { and } \quad Q:=S_{h, k}\left(\mathcal{T}_{h}\right),
$$

where we note that the polynomial degree employed in $\boldsymbol{V}$ must be one degree higher than that in $Q$ in order to obtain a stable approximation see e.g. [16] and references therein for further discussion regarding stables choices of FE space for the approximation of Stokes flow.

Prior to specifying the finite element method used for the microscale system, we highlight that the tensor system may be decomposed into $d$ standard (vector) Stokes problems. Therefore, it is sufficient to consider the discretization of a standard Stokes problem, subject to appropriate stress, slip and penetration conditions on $\Gamma$ obtained from (8) by considering the interface conditions for the tensor problem in a componentwise manner. As such, we may write the Stokes problem for the $i^{\text {th }}$ column of $\boldsymbol{K}$, denoted $\boldsymbol{\Phi}$, and the $i^{\text {th }}$ component of $\boldsymbol{a}$, denoted $\phi$, for $1 \leqslant i \leqslant d$, as

$$
\left.\begin{array}{rlrl}
\nabla_{\boldsymbol{x}} \phi-\mu \nabla_{\boldsymbol{x}} \cdot \mathbf{D}_{\boldsymbol{x}}(\boldsymbol{\Phi}) & =\boldsymbol{f}_{i} & & \forall \boldsymbol{x} \in \Omega_{\ell}, \\
\nabla_{\boldsymbol{x}} \cdot \boldsymbol{\Phi} & =0 & & \forall \boldsymbol{x} \in \Omega_{\ell},
\end{array}\right\}
$$

where $\left(\boldsymbol{f}_{i}\right)_{j}=\delta_{i j}$, subject to the following conditions on the interface $\Gamma$

$$
\left.\begin{array}{rlrl}
{\left[\phi \boldsymbol{n}_{\Gamma}-\mu \boldsymbol{D}_{\boldsymbol{x}}(\boldsymbol{\Phi}) \boldsymbol{n}_{\Gamma}\right]_{-}^{+}=\mathbf{0}} & & \forall \boldsymbol{x} \in \Gamma, \\
{\left[\boldsymbol{\Phi} \cdot \boldsymbol{\tau}_{\Gamma}\right]_{-}^{+}=0} & \forall \boldsymbol{x} \in \Gamma, \\
\left(\boldsymbol{\Phi} \cdot \boldsymbol{n}_{\Gamma}\right)^{+}=0 & \forall \boldsymbol{x} \in \Gamma, \\
\left(\boldsymbol{\Phi} \cdot \boldsymbol{n}_{\Gamma}\right)^{-}=0 & \forall \boldsymbol{x} \in \Gamma,
\end{array}\right\}
$$

and periodic boundary conditions on $\partial \Omega_{\ell}$ for all components of $\boldsymbol{\Phi}$ and all components of $\phi \boldsymbol{n}_{\Gamma}-\mu \boldsymbol{D}_{\boldsymbol{x}}(\mathbf{\Phi}) \boldsymbol{n}_{\Gamma}$. As in the tensor formulation, we impose that $\phi$ 
is mean-free in order to guarantee its uniqueness, i.e.

$$
\int_{\Omega_{\ell}} \phi d x=0
$$

To obtain the dG FE discretization we first multiply (31) by test functions $(\boldsymbol{\Psi}, \psi)$, yielding the variational formulation

$$
\left.\begin{array}{rlrl}
\int_{\Omega_{\ell}}\left(\nabla_{\boldsymbol{x}} \phi-\mu \nabla_{\boldsymbol{x}} \cdot \boldsymbol{D}_{\boldsymbol{x}}(\boldsymbol{\Phi})\right) \cdot \boldsymbol{\Psi} d \boldsymbol{x} & =\int_{\Omega_{\ell}} \boldsymbol{f}_{i} \cdot \boldsymbol{\Psi} d \boldsymbol{x} & & \forall \boldsymbol{\Psi}, \\
\int_{\Omega_{\ell}} \nabla_{\boldsymbol{x}} \cdot \boldsymbol{\Phi} \psi d \boldsymbol{x} & =0 & \forall \psi .
\end{array}\right\}
$$

We then apply the integration by parts formulae

$$
\int_{\kappa} \nabla_{\boldsymbol{x}} \phi \cdot \boldsymbol{\Psi} d \boldsymbol{x}=-\int_{\kappa} \phi \nabla_{\boldsymbol{x}} \cdot \boldsymbol{\Psi} d \boldsymbol{x}+\int_{\partial \kappa} \phi \boldsymbol{\Psi} \cdot \boldsymbol{n}_{\kappa} d s
$$

and

$$
\int_{\kappa} \nabla_{\boldsymbol{x}} \cdot \boldsymbol{D}_{\boldsymbol{x}}(\boldsymbol{\Phi}) \cdot \boldsymbol{\Psi} d \boldsymbol{x}=-\int_{\kappa} \boldsymbol{D}_{\boldsymbol{x}}(\boldsymbol{\Phi}): \boldsymbol{D}_{\boldsymbol{x}}(\boldsymbol{\Psi}) d \boldsymbol{x}+\int_{\partial \kappa} \boldsymbol{D}_{\boldsymbol{x}}(\boldsymbol{\Phi}): \boldsymbol{\Psi} \otimes \boldsymbol{n}_{\kappa} d s
$$

(from [44]) elementwise, making an appropriate assumption on numerical fluxes. We then introduce appropriate penalization terms for the velocity space in order to weakly impose inter-element continuity and the velocity interface conditions, as in [44]. As such, we propose the dG FE method given by: find $\left(\boldsymbol{\Phi}_{h, \boldsymbol{k}}, \phi_{h, \boldsymbol{k}}\right) \in$ $\boldsymbol{V} \times Q$ such that

$$
\begin{array}{ccccc}
a\left(\boldsymbol{\Phi}_{h, \boldsymbol{k}}, \boldsymbol{\Psi}\right)+b\left(\boldsymbol{\Psi}, \phi_{h, \boldsymbol{k}}\right) & = & \left(\boldsymbol{f}_{i}, \boldsymbol{\Psi}\right)_{\left(L^{2}\left(\Omega_{\ell}\right)\right)^{d}} & & \forall \boldsymbol{\Psi} \in \boldsymbol{V}, \\
b\left(\boldsymbol{\Phi}_{h, \boldsymbol{k}}, \psi\right) & = & 0 & & \forall \psi \in Q, \\
c_{\Gamma}\left(\boldsymbol{\Psi}, \boldsymbol{\Phi}_{h, \boldsymbol{k}}, \phi_{h, \boldsymbol{k}}\right) & = & 0 & & \forall \boldsymbol{\Psi} \in \boldsymbol{V},
\end{array}
$$

for $1 \leqslant i \leqslant d$, where $\boldsymbol{k}$ denotes the polynomial degrees used in $\boldsymbol{V} \times Q$, $(\cdot, \cdot)_{\left(L^{2}\left(\Omega_{\ell}\right)\right)^{d}}$ denotes the inner product on $\left(L^{2}\left(\Omega_{\ell}\right)\right)^{d}$, and the bilinear forms $a(\cdot, \cdot), b(\cdot, \cdot)$, and trilinear form $c_{\Gamma}(\cdot, \cdot, \cdot)$ are defined by

$$
\begin{aligned}
& a(\boldsymbol{v}, \boldsymbol{w}):=\sum_{\kappa \in \mathcal{T}_{h}} \int_{\kappa} \mu \boldsymbol{D}_{\boldsymbol{x}}(\boldsymbol{v}): \boldsymbol{D}_{\boldsymbol{x}}(\boldsymbol{w}) d \boldsymbol{x}-\sum_{F \in \mathcal{E}\left(\mathcal{T}_{h}\right)} \int_{F} \mu\left\{\left\{\boldsymbol{D}_{\boldsymbol{x}}(\boldsymbol{v})\right\}: \underline{\llbracket \boldsymbol{w} \rrbracket} d s\right. \\
& -\sum_{F \in \mathcal{E}\left(\mathcal{T}_{h}\right)} \int_{F} \mu\left\{\boldsymbol{D}_{\boldsymbol{x}}(\boldsymbol{w})\right\}: \underline{\llbracket \boldsymbol{v} \rrbracket} d s+\sum_{F \in \mathcal{E}\left(\mathcal{T}_{h}\right)} \int_{F} \vartheta_{1} \mu \llbracket \boldsymbol{v} \rrbracket: \underline{\llbracket \boldsymbol{w} \rrbracket} d s, \\
& b(\boldsymbol{v}, q):=\sum_{k \in \mathcal{T}_{h}} \int_{\kappa}-q\left(\nabla_{\boldsymbol{x}} \cdot \boldsymbol{v}\right) d \boldsymbol{x}+\sum_{F \in \mathcal{E}\left(\mathcal{T}_{h}\right)} \int_{F}\{q q \rrbracket \llbracket \boldsymbol{v} \rrbracket d s,
\end{aligned}
$$




$$
\begin{aligned}
c_{\Gamma}(\boldsymbol{v}, \boldsymbol{w}, q):= & -\sum_{F \in \mathcal{E}_{\Gamma}\left(\mathcal{T}_{h}\right)} \int_{F} \mu\left\{\boldsymbol{D}_{\boldsymbol{x}}(\boldsymbol{w})\right\}: \underline{\llbracket v \rrbracket}+\mu\left\{\boldsymbol{D}_{\boldsymbol{x}}(\boldsymbol{v})\right\}: \underline{\llbracket \boldsymbol{w} \rrbracket}-\{q\} \llbracket \boldsymbol{v} \rrbracket d s+ \\
& \sum_{F \in \mathcal{E}_{\Gamma}\left(\mathcal{T}_{h}\right)} \int_{F} \vartheta_{2} \mu\left(\left(\left.\boldsymbol{w}\right|_{\Omega_{1}} \cdot \boldsymbol{n}_{\kappa}\right)\left(\left.\boldsymbol{v}\right|_{\Omega_{1}} \cdot \boldsymbol{n}_{\kappa}\right)+\left(\left.\boldsymbol{w}\right|_{\Omega_{2}} \cdot \boldsymbol{n}_{\kappa}\right)\left(\left.\boldsymbol{v}\right|_{\Omega_{2}} \cdot \boldsymbol{n}_{\kappa}\right)\right) d s+ \\
& \sum_{F \in \mathcal{E}_{\Gamma}\left(\mathcal{T}_{h}\right)} \int_{F} \vartheta_{2} \mu \llbracket \boldsymbol{w} \cdot \boldsymbol{\tau}_{\kappa} \rrbracket \llbracket \boldsymbol{v} \cdot \boldsymbol{\tau}_{\kappa} \rrbracket d s,
\end{aligned}
$$

for $\boldsymbol{v}, \boldsymbol{w} \in\left(X\left(\mathcal{T}_{h}\right)\right)^{d}$ and $q \in X\left(\mathcal{T}_{h}\right)$, where $X\left(\mathcal{T}_{h}\right)$ denotes the broken $H^{1}(\Omega)$ Sobolev space defined by

$$
X\left(\mathcal{T}_{h}\right):=\left\{v \in L^{2}\left(\Omega_{\ell}\right): v \in H^{1}(\kappa) \forall \kappa \in \mathcal{T}_{h}\right\},
$$

and $\vartheta_{i}$ denotes a penalization parameter defined by

$$
\vartheta_{i}:=C_{P}^{i} \frac{k^{2}}{h} \quad \text { for } i=1,2,
$$

for some suitably large parameter $C_{P}^{i}$ that is independent of $h$ and $k$. The choice of penalization parameter here is motivated by that made in [44], in which a parameter of this form is employed to obtain optimal rates of convergence for a classical Stokes problem. We note that $a(\cdot, \cdot)$ and $b(\cdot, \cdot)$ are equivalent to the bilinear forms presented in [44]; however, we introduce the additional term $c_{\Gamma}(\cdot, \cdot, \cdot)$ here as a means of enforcing the interface condition on $\Gamma$. While there are many means of imposing the interface conditions, such as the transition region approach proposed for Stokes-Darcy coupling in [20, 21], we adopt here the form of $c_{\Gamma}$ given in (40) as it arises naturally from the stress, slip, and penetration conditions (32) when deriving the dG FE method.

In order to impose the mean-free condition (33), we fix the value of a single degree of freedom in the linear system, prior to solving. After the computation of the solution to the linear system, we subsequently subtract the mean of the computed (non-mean-free) FE approximation, to yield our final mean-free approximation.

\subsection{Discretization of the Macroscale System}

In the discretization of the macroscale system we adopt analogous notation to that introduced in section 3.1. However, we now restrict our attention to a 
two-dimensional domain $\Omega_{L}$ for purely illustrative purposes, and consider only shape-regular triangulations, $\mathcal{T}_{H}$. By $H_{\kappa}$ we denote the element diameter of $\kappa \in \mathcal{T}_{H}$, and define the mesh function $H:=\max \left\{H_{\kappa}: \kappa \in \mathcal{T}_{H}\right\}$. Note, however, that for convenience there is a minor abuse of notation in what follows, as we let $k$ denote the polynomial degree of multiple FE spaces, in addition to those defined in section 3.1. For the FE spaces used on the macroscale there is no reason to consider them equal and for the numerical experiments presented in section 4 we shall specify a particular value for each FE space.

\subsubsection{Flow}

We now consider the discretization of the macroscale flow system given by (13) and (14). To this end, we introduce the Raviart-Thomas (RT) element $[30,38]$ on shape regular simplices given by

$$
R T_{k}(\kappa):=\left(\mathcal{P}_{k}(\kappa)\right)^{d}+\boldsymbol{x} \mathcal{P}_{k}(\kappa)
$$

for $d=2,3$. It is possible to show, see e.g. [16], that functions $\boldsymbol{v} \in R T_{k}(\kappa)$ may be fully characterized by the moments of up to order $k$ of $\boldsymbol{v} \cdot \boldsymbol{n}_{\kappa}$ on the edges of $\kappa$ and the moments of up to order $k-1$ of $\boldsymbol{v}$ on the interior of $\kappa$. Given this, we may construct the RT FE space

$$
R T_{k}\left(\Omega_{L}, \mathcal{T}_{H}\right):=\left\{\boldsymbol{v} \in H\left(\operatorname{div}, \Omega_{L}\right):\left.\boldsymbol{v}\right|_{\kappa} \in R T_{k}(\kappa) \quad \forall \kappa \in \mathcal{T}_{H}\right\}
$$

where $H\left(\operatorname{div}, \Omega_{L}\right)$ is the Sobolev space

$$
H\left(\operatorname{div}, \Omega_{L}\right):=\left\{\boldsymbol{v} \in\left(L^{2}\left(\Omega_{L}\right)\right)^{d}: \nabla_{\boldsymbol{X}} \cdot \boldsymbol{v} \in L^{2}\left(\Omega_{L}\right)\right\}
$$

Remark 1. The choice of a $H\left(\right.$ div,$\left.\Omega_{L}\right)$-conforming FE space ensures that there is no unphysical compression or rarefaction associated with a lack of continuity of the normal trace of the velocity across element boundaries when computing the FE approximation to the solution of the hyperbolic transport equations for drug, nutrient and mixture component volume fraction. We highlight that alternative choices of $H\left(\right.$ div,$\left.\Omega_{L}\right)$-conforming elements, such as BDM [13, 15] or BDFM [14], may also be employed. 
Thereby, setting

$$
\mathcal{V}:=R T_{k}\left(\Omega_{L}, \mathcal{T}_{H}\right) \quad \text { and } \quad \mathcal{Q}:=S_{H, k}\left(\mathcal{T}_{H}\right)
$$

the FE approximation of the macroscale flow problem, given in (13)-(14), is defined by: find $\left(\boldsymbol{u}_{H, k}, p_{H, k}\right) \in \mathcal{V} \times \mathcal{Q}$ such that

$$
\left.\begin{array}{lll}
A_{F}\left(\boldsymbol{u}_{H, k}, \boldsymbol{v}\right)+B_{F}\left(\boldsymbol{v}, p_{H, k}\right) & =G_{F}(\boldsymbol{v}) & \forall \boldsymbol{v} \in \mathcal{V}, \\
B_{F}\left(\boldsymbol{u}_{H, k}, q\right) & =L_{F}(q ; \boldsymbol{\theta}) & \forall q \in \mathcal{Q} .
\end{array}\right\}
$$

The bilinear forms $A_{F}(\cdot, \cdot)$ and $B_{F}(\cdot, \cdot)$ are defined as

$$
A_{F}(\boldsymbol{v}, \boldsymbol{w}):=\int_{\Omega_{L}}\langle\boldsymbol{K}\rangle_{\Omega}^{-1} \boldsymbol{v} \cdot \boldsymbol{w} d \boldsymbol{X}, \quad \boldsymbol{v}, \boldsymbol{w} \in H\left(\operatorname{div}, \Omega_{L}\right),
$$

and

$$
B_{F}(\boldsymbol{v}, q):=-\int_{\Omega_{L}} q \nabla_{\boldsymbol{X}} \cdot \boldsymbol{v} d \boldsymbol{X}, \quad \boldsymbol{v} \in H\left(\operatorname{div}, \Omega_{L}\right), q \in L^{2}\left(\Omega_{L}\right),
$$

respectively, and the linear functionals $G_{F}(\cdot)$ and $L_{F}(\cdot ; \boldsymbol{\theta})$ are defined as

$$
G_{F}(\boldsymbol{v}):=-\left\langle\boldsymbol{v} \cdot \boldsymbol{n}, p_{D}\right\rangle_{\partial \Omega_{L}}, \quad \boldsymbol{v} \in H\left(\operatorname{div}, \Omega_{L}\right)
$$

and

$$
L_{F}(q ; \boldsymbol{\theta}):=-(f(\boldsymbol{X} ; \boldsymbol{\theta}), q)_{L^{2}\left(\Omega_{L}\right)}, \quad q \in L^{2}\left(\Omega_{L}\right),
$$

where $\langle\cdot, \cdot\rangle_{\partial \Omega_{L}}$ denotes the duality pairing between $H^{\frac{1}{2}}\left(\partial \Omega_{L}\right)$ and $H^{-\frac{1}{2}}\left(\partial \Omega_{L}\right)$, and $(\cdot, \cdot)_{L^{2}\left(\Omega_{L}\right)}$ denotes the inner product on $L^{2}\left(\Omega_{L}\right)$.

\subsubsection{Semi-Discrete Approximation of Transport Equations (15), (19), and}

The discretization presented in this section follows closely the dG FE method presented in [26]. Given this, in addition to the notation introduced in the previous sections, for an element $\kappa \in \mathcal{T}_{H}$, we define the inflow and outflow parts of the element boundary $\partial \kappa$ by

$$
\partial^{-} \kappa(\boldsymbol{b}):=\left\{\boldsymbol{X} \in \partial \kappa: \boldsymbol{b}(\boldsymbol{X}) \cdot \boldsymbol{n}_{\kappa}<0\right\}
$$

and

$$
\partial^{+} \kappa(\boldsymbol{b}):=\left\{\boldsymbol{X} \in \partial \kappa: \boldsymbol{b}(\boldsymbol{X}) \cdot \boldsymbol{n}_{\kappa}>0\right\},
$$


for a given vector field $\boldsymbol{b}$, and recalling that $\boldsymbol{n}_{\kappa}$ is the outward facing normal to $\kappa \in \mathcal{T}_{H}$. We now extend the \pm notation introduced in section 2.2 to describe the interior and exterior traces of a function $q$ on $\mathcal{T}_{H}$. As such, for an element $\kappa \in \mathcal{T}_{H}$ we define $q^{+}$and $q^{-}$to be the interior and exterior traces of $q$ on some face $F \in \mathcal{E}_{I}\left(\mathcal{T}_{H}\right)$ of $\kappa$, respectively. Then for each component of the mixture $1 \leqslant i \leqslant N_{\theta}$ governed by (15)-(18), we specify the semi-discrete approximation $\theta_{H, k, i}(\cdot, t) \in \mathcal{Q}$ as the solution of

$$
\begin{aligned}
&\left(\frac{\partial \theta_{H, k, i}}{\partial t}, q\right)_{L^{2}\left(\Omega_{L}\right)}+B_{a}\left(\theta_{H, k, i}, q ; \tilde{\boldsymbol{u}}_{H, k}\right)+B_{r}\left(\theta_{H, k, i}, q ; \mathcal{R}_{\theta, i}\right) \\
&=L_{S}\left(q ; g_{\theta, i}\right)+L_{\partial}\left(q ; \tilde{\boldsymbol{u}}_{H, k}, \boldsymbol{\Theta}\right) \quad \forall q \in \mathcal{Q},
\end{aligned}
$$

wherein we have employed the FE approximation of the velocity, utilizing an equivalent discrete definition for $\tilde{\boldsymbol{u}}_{H, k}$ to that in (16) for the continuous case, $\Theta$ is the boundary data defined in (17), and we define the bilinear/semilinear forms $B_{a}(\cdot, \cdot ; \cdot)$ and $B_{r}(\cdot, \cdot ; \cdot)$ by

$$
\begin{array}{r}
B_{a}(w, v ; \boldsymbol{b}):=\sum_{\kappa \in \mathcal{T}_{H}}\left(\int_{\kappa} v \boldsymbol{b} \cdot \nabla_{\boldsymbol{X}} w d \boldsymbol{X}-\int_{\partial^{-} \kappa(\boldsymbol{b}) \backslash \partial \Omega_{L}}\left(\boldsymbol{b} \cdot \boldsymbol{n}_{\kappa}\right)\left(w^{+}-w^{-}\right) v^{+} d S-\right. \\
\left.\int_{\partial^{-} \kappa(\boldsymbol{b}) \cap \partial \Omega_{L}}\left(\boldsymbol{b} \cdot \boldsymbol{n}_{\kappa}\right) w^{+} v^{+} d S\right),
\end{array}
$$

and

$$
B_{r}(w, v ; \mathcal{R}):=\sum_{\kappa \in \mathcal{T}_{H}} \int_{\kappa} \mathcal{R} w v d \boldsymbol{X}
$$

respectively, and the linear functionals $L_{s}(\cdot ; \cdot)$ and $L_{\partial}(\cdot ; \cdot, \cdot)$ by

$$
L_{S}(v ; g):=\sum_{\kappa \in \mathcal{T}_{H}} \int_{\kappa} g v d \boldsymbol{X},
$$

and

$$
L_{\partial}(v ; \boldsymbol{b}, g):=\sum_{\kappa \in \mathcal{T}_{h}} \int_{\partial^{-} \kappa(\boldsymbol{b}) \cap \partial \Omega_{L}}-\left(\boldsymbol{b} \cdot \boldsymbol{n}_{\kappa}\right) g v^{+} d S,
$$


for $w, v \in X\left(\mathcal{T}_{H}\right)$, cf. [26]. Similarly, for the transport of drug, governed by (19)(21), we define the semi-discrete approximation $c_{H, k}(\cdot, t) \in \mathcal{Q}$ as the solution of

$$
\begin{aligned}
\left(\frac{\partial c_{H, k}}{\partial t}, q\right)_{L^{2}\left(\Omega_{L}\right)}+B_{a}\left(c_{H, k}, q ; \boldsymbol{u}_{H, k}\right)+ & B_{r}\left(c_{H, k}, q ; \mathcal{R}_{c}\right) \\
& =L_{\partial}\left(q ; \boldsymbol{u}_{H, k}, C\right) \quad \forall q \in \mathcal{Q},
\end{aligned}
$$

and for the transport of nutrient, governed by (22)-(24), we define the semidiscrete approximation $n_{H, k}(\cdot, t) \in \mathcal{Q}$ as the solution of

$$
\begin{aligned}
\left(\frac{\partial n_{H, k}}{\partial t}, q\right)_{L^{2}\left(\Omega_{L}\right)}+B_{a}\left(n_{H, k}, q ; \boldsymbol{u}_{H, k}\right)+ & B_{r}\left(n_{H, k}, q ; \mathcal{R}_{n}\right) \\
& =L_{\partial}\left(q ; \boldsymbol{u}_{H, k}, N\right) \quad \forall q \in \mathcal{Q} .
\end{aligned}
$$

\subsubsection{Fully-discrete Approximation}

We consider now the fully-discrete approximation of the macroscale velocity, pressure, drug and nutrient concentrations, and mixture component volume fractions for $t \in[0, T]$. We denote the (uniform) time-step size by $\Delta t$ and the function $v$ at time $m \Delta t$ by $v^{m}$. For the granularity of the spatial meshes utilized in the numerical experiments presented in section 4.2 , the stability limits on the time-step associated with an explicit scheme mean that the error incurred from the spatial discretization dominates the temporal error, even for a first order explicit Euler scheme. As such, we employ an explicit Euler discretization to form the fully-discrete approximation.

We first project the initial conditions for $c, n$, and $\boldsymbol{\theta}$ onto the appropriate finite element spaces by solving: find $c_{H, k}^{0} \in \mathcal{Q}, n_{H, k}^{0} \in \mathcal{Q}$, and $\boldsymbol{\theta}_{H, k}^{0} \in \mathcal{Q}^{N_{\theta}}$ such that

$$
\begin{array}{ll}
\left(c_{H, k}^{0}-C_{0}, q\right)_{L^{2}\left(\Omega_{L}\right)}=0 & \forall q \in \mathcal{Q} \\
\left(n_{H, k}^{0}-N_{0}, q\right)_{L^{2}\left(\Omega_{L}\right)}=0 & \forall q \in \mathcal{Q}
\end{array}
$$

and for each component of the mixture $1 \leqslant i \leqslant N_{\theta}$,

$$
\left(\theta_{H, k, i}^{0}-\Theta_{i}, q\right)_{L^{2}\left(\Omega_{L}\right)}=0 \quad \forall q \in \mathcal{Q} .
$$


We may then solve the initial macroscale flow problem (cf. (47)): find $\left(\boldsymbol{u}_{H, k}^{0}, p_{H, k}^{0}\right) \in \mathcal{V} \times \mathcal{Q}$ such that

$$
\left.\begin{array}{llcl}
A_{F}\left(\boldsymbol{u}_{H, k}^{0}, \boldsymbol{v}\right)+B_{F}\left(\boldsymbol{v}, p_{H, k}^{0}\right) & = & 0 & \forall \boldsymbol{v} \in \mathcal{V}, \\
B_{F}\left(\boldsymbol{u}_{H, k}^{0}, q\right) & =L_{F}\left(q ; \boldsymbol{\theta}_{H, k}^{0}\right) & & \forall q \in \mathcal{Q} .
\end{array}\right\}
$$

Then, for $0 \leqslant m \leqslant M-1$, we solve the transport problems for drug, nutrient,

and the components of the mixture: find $c_{H, k}^{m+1} \in \mathcal{Q}, n_{H, k}^{m+1} \in \mathcal{Q}$, and $\boldsymbol{\theta}_{H, k}^{m+1} \in \mathcal{Q}^{N_{\theta}}$ such that

$$
\begin{array}{r}
\left(\frac{c_{H, k}^{m+1}-c_{H, k}^{m}}{\Delta t}, q\right)_{L^{2}\left(\Omega_{L}\right)}+B_{a}\left(c_{H, k}^{m}, q ; \boldsymbol{u}_{H, k}^{m}\right)+B_{r}\left(c_{H, k}^{m}, q ; \mathcal{R}_{c}^{m}\right) \\
=L_{\partial}\left(q ; \boldsymbol{u}_{H, k}^{m}, C^{m}\right) \quad \forall q \in \mathcal{Q}, \\
\left(\frac{n_{H, k}^{m+1}-n_{H, k}^{m}}{\Delta t}, q\right)_{L^{2}\left(\Omega_{L}\right)}+B_{a}\left(n_{H, k}^{m}, q ; \boldsymbol{u}_{H, k}^{m}\right)+B_{r}\left(n_{H, k}^{m}, q ; \mathcal{R}_{n}^{m}\right) \\
=L_{\partial}\left(q ; \boldsymbol{u}_{H, k}^{m}, N^{m}\right) \quad \forall q \in \mathcal{Q},
\end{array}
$$

and for each component of the mixture $1 \leqslant i \leqslant N_{\theta}$,

$$
\begin{aligned}
\left(\frac{\theta_{H, k, i}^{m+1}-\theta_{H, k, i}^{m}}{\Delta t}, q\right)_{L^{2}\left(\Omega_{L}\right)} & +B_{a}\left(\theta_{H, k, i}^{m}, q ; \tilde{\boldsymbol{u}}_{H, k}^{m}\right)+B_{r}\left(\theta_{H, k, i}^{m}, q ; \mathcal{R}_{\theta, i}^{m}\right) \\
= & L_{s}\left(q ; g_{\theta, i}^{m}\right)+L_{\partial}\left(q ; \tilde{\boldsymbol{u}}_{H, k}^{m}, \Theta_{i}^{m}\right) \quad \forall q \in \mathcal{Q} .
\end{aligned}
$$

We may then solve the flow problem: find $\left(\boldsymbol{u}_{H, k}^{m+1}, p_{h, k}^{m+1}\right) \in \mathcal{V} \times \mathcal{Q}$ such that

$$
\left.\begin{array}{llcc}
A_{F}\left(\boldsymbol{u}_{H, k}^{m+1}, \boldsymbol{v}\right)+B_{F}\left(\boldsymbol{v}, p_{H, k}^{m+1}\right) & = & 0 & \forall \boldsymbol{v} \in \mathcal{V}, \\
B_{F}\left(\boldsymbol{u}_{H, k}^{m+1}, q\right) & =L_{F}\left(q ; \boldsymbol{\theta}_{H, k}^{m}\right) & \forall q \in \mathcal{Q} .
\end{array}\right\}
$$

Algorithm 3.1 sets out the solution procedure for the numerical approximation of the macroscale system.

\section{Numerical Experiments}

In this section we present a series of representative numerical examples to demonstrate the implementation of the discretizations described in section 3 . 


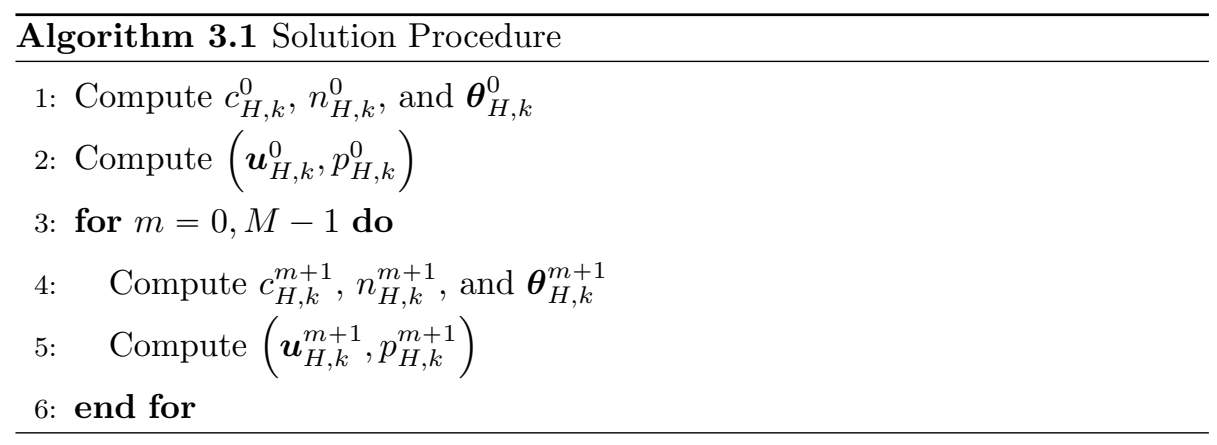

The computations presented in the section have been undertaken using the AptoFEM package [7] with the MUMPS linear solver $[5,6]$. We also note that all unstructured meshes presented in this section were generated using the Triangle mesh generator [40].

Remark 2. While MUMPS works well for the two-dimensional computations presented here, as well as the three-dimensional computations employed in the parameterisation of the macroscale examples, we remark that this will typically not be the case for microscale flow problems solved in complex pore structures. As such, it is likely that the use of a suitably preconditioned iterative method will be required (though the provision of such methods are beyond the scope of this work).

\subsection{Microscale}

In order to verify the implementation of the discretization presented in section 3.1 we present two numerical experiments that demonstrate the convergence of the FE approximation. In Example 1 we consider a problem that has a known analytical solution, so that we may study the rate of convergence of the FE approximation with respect to the granularity of the mesh, for a range of polynomial degrees. In Example 2, we consider a more complicated domain for which an analytical solution is not available. In [18], we consider three-dimensional domains in order to obtain solutions that result in non-zero spatially-averaged permeabilities in both subdomains. Here, however, we consider two-dimensional problems so that we are able to make quantitative 
statements regarding the analytical solution and demonstrate convergence of quantities used in the parameterization of the macroscale system.

\section{Example 1}

Instead of the periodic boundary conditions described in section 2.2, we consider a problem with a Dirichlet boundary condition on some portion of the boundary $\Gamma_{D}$, a Neumann condition on $\Gamma_{N}=\partial \Omega_{\ell} \backslash \Gamma_{D}$, and remove the requirement that $\phi$ is mean-free. As such, we adapt the discretization described in section 3.1 to incorporate appropriate terms for the imposition of these boundary conditions, thus obtaining the finite element problem: find $\left(\boldsymbol{\Phi}_{h, \boldsymbol{k}}, \phi_{h, \boldsymbol{k}}\right) \in \boldsymbol{V} \times Q$ such that

$$
\left.\begin{array}{cccc}
a\left(\boldsymbol{\Phi}_{h, \boldsymbol{k}}, \boldsymbol{\Psi}\right)+b\left(\boldsymbol{\Psi}, \phi_{h, \boldsymbol{k}}\right) & =\hat{f}(\boldsymbol{\Psi}) & & \forall \boldsymbol{\Psi} \in \boldsymbol{V}, \\
b\left(\boldsymbol{\Phi}_{h, \boldsymbol{k}}, \psi\right) & =\hat{g}(\psi) & & \forall \psi \in Q, \\
c_{\Gamma}\left(\boldsymbol{\Psi}, \boldsymbol{\Phi}_{h, \boldsymbol{k}}, \phi_{h, \boldsymbol{k}}\right) & =0 & & \forall \boldsymbol{\Psi} \in \boldsymbol{V},
\end{array}\right\}
$$

where $a, b$, and $c_{\Gamma}$ are as defined in (38)-(40), and the linear functionals $\hat{f}$ and $\hat{g}$ are given by

$$
\begin{aligned}
& \hat{f}(\boldsymbol{\Psi})=(\boldsymbol{f}, \boldsymbol{\Psi})_{\left(L^{2}\left(\Omega_{\ell}\right)\right)^{d}}+ \\
& \sum_{F \in \mathcal{E}\left(\mathcal{T}_{h}\right)}\left(\int_{F \cap \Gamma_{D}}\left(-\mu \boldsymbol{D}_{\boldsymbol{x}}(\boldsymbol{\Psi}): \boldsymbol{g}_{D} \otimes \boldsymbol{n}_{\boldsymbol{\kappa}}+\mu \vartheta_{1} \boldsymbol{g}_{D} \cdot \boldsymbol{\Psi}\right) d S+\int_{F \cap \Gamma_{N}} \boldsymbol{g}_{N} \cdot \boldsymbol{\Psi} d s\right),
\end{aligned}
$$

and

$$
\hat{g}(\psi)=\sum_{F \in \mathcal{E}\left(\mathcal{T}_{h}\right)} \int_{F \cap \Gamma_{N}} \psi\left(\boldsymbol{g}_{D} \cdot \boldsymbol{n}_{\boldsymbol{\kappa}}\right) d s,
$$

where $\boldsymbol{g}_{D}$ and $\boldsymbol{g}_{N}$ denote the Dirichlet and Neumann data, respectively. We proceed by defining the geometry as $\Omega_{1}=(0,0.5) \times(0,1), \Omega_{2}=(0.5,1) \times(0,1)$, $\Omega_{\ell}=\Omega_{1} \cup \Omega_{2}$, and $\Gamma_{N}=(0,1) \times\{1\}$. We further specify that the boundary and forcing data are given such that

$$
\boldsymbol{\Phi}=\left(\begin{array}{c}
0 \\
\sin \left(\pi x_{1}\right)
\end{array}\right) \quad \text { and } \quad \phi=1-x_{2},
$$




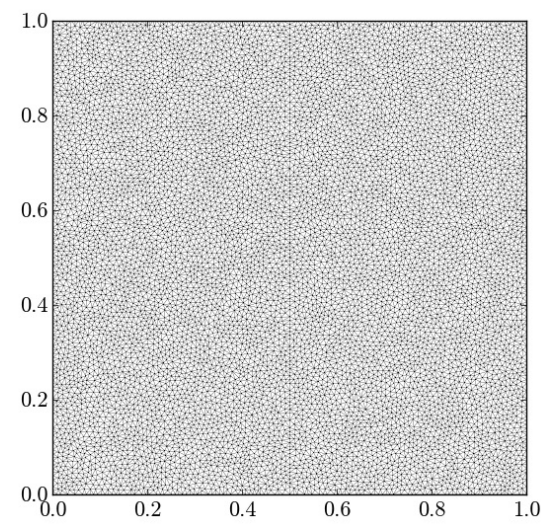

Figure 3: A representative mesh, $\mathcal{T}_{h}$.

i.e.

$$
\boldsymbol{f}=\left(\begin{array}{c}
0 \\
1+\pi^{2} \sin \left(\pi x_{1}\right)
\end{array}\right) \text { and } \boldsymbol{g}_{N}=\left(\begin{array}{c}
\pi \cos \left(\pi x_{1}\right) \\
1-x_{2}
\end{array}\right) .
$$

Figure 4 shows a representative FE solution for the microscale system obtained by employing the mesh shown in Figure 3. Figure 5 demonstrates rates of convergence of the numerical approximation with respect to the mesh granularity and polynomial degree for a selection of norms. For the smooth analytical solution considered in this example, we observe optimal rates of convergence of $\boldsymbol{\Phi}_{h, \boldsymbol{k}}$ and $\phi_{h, \boldsymbol{k}}$ in the appropriate $L^{2}\left(\Omega_{\ell}\right)$ norm, as shown in Figures 5(a) and 5(b), respectively. Similarly, in Figures 5(c) and 5(d) we observe at least $\mathcal{O}\left(h^{k+2}\right)$ convergence for the slip and penetration interface conditions, given by (8), in the $L^{2}(\Gamma)$ norm.

\section{Example 2}

In this example we now impose periodic boundary conditions as described in section 2.2, the mean-free constraint (33), and solve the FE problem as prescribed in (37) for forcing data

$$
f=\left(\begin{array}{c}
0 \\
1
\end{array}\right)
$$




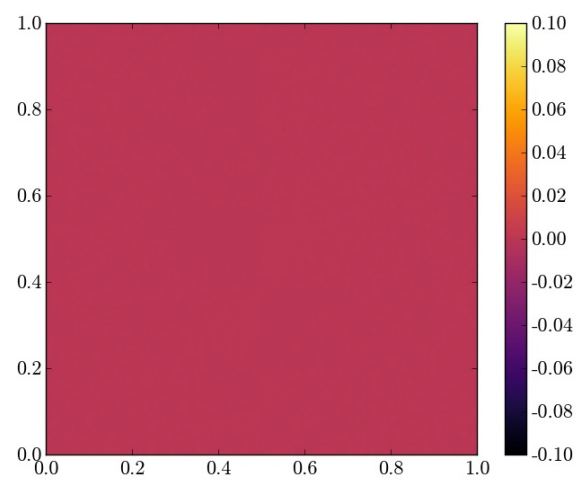

(a) $\left(\boldsymbol{\Phi}_{h, \boldsymbol{k}}\right)_{1}$

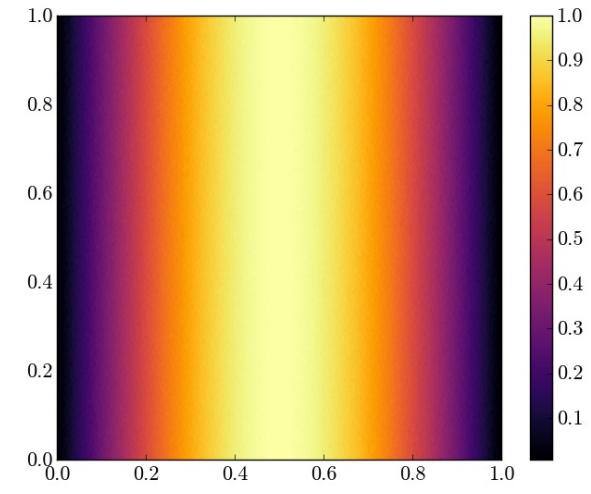

(b) $\left(\boldsymbol{\Phi}_{h, \boldsymbol{k}}\right)_{2}$.

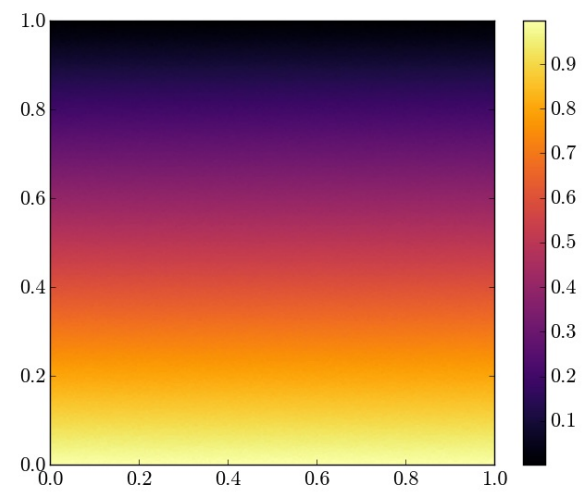

(c) $\phi_{h, k}$.

Figure 4: The FE approximation $\left(\boldsymbol{\Phi}_{h, \boldsymbol{k}}, \phi_{h, \boldsymbol{k}}\right)$ for Example 1, obtained employing the mesh shown in Figure 3 with polynomial degree $\boldsymbol{k}=(2,2,1)$. 


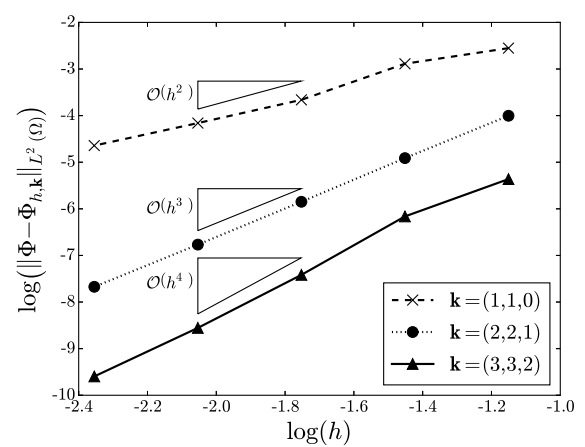

(a) Convergence of the $L^{2}\left(\Omega_{\ell}\right)$ error of $\boldsymbol{\Phi}_{h, \boldsymbol{k}}$.

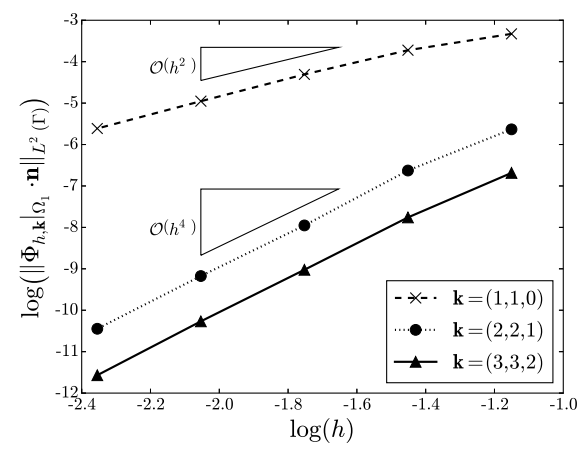

(c) Convergence of the $L^{2}(\Gamma)$ error of the no-penetration condition across $\Gamma$, in $\Omega_{1}$.

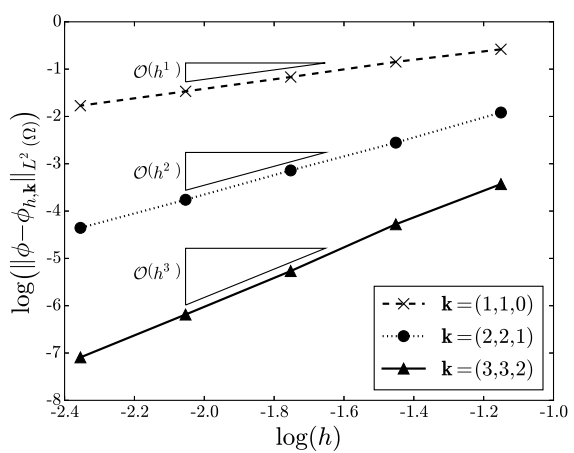

(b) Convergence of the $L^{2}\left(\Omega_{\ell}\right)$ error of $\phi_{h, k}$.

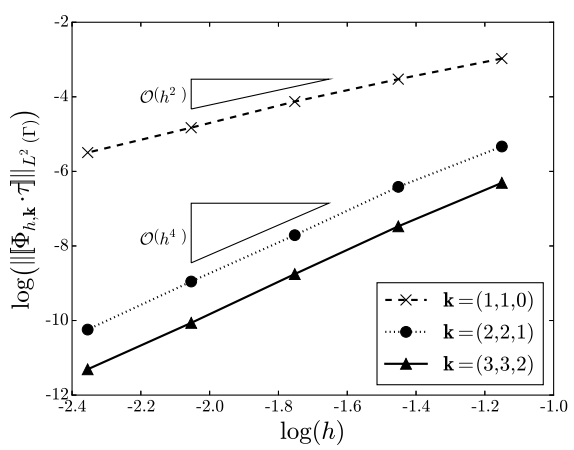

(d) Convergence of the $L^{2}(\Gamma)$ error of the slip condition on $\Gamma$.

Figure 5: Plots demonstrating the rate of convergence of the FE approximation $\left(\boldsymbol{\Phi}_{h, \boldsymbol{k}}, \phi_{h, \boldsymbol{k}}\right)$ in Example 1 for a selection of norms. 


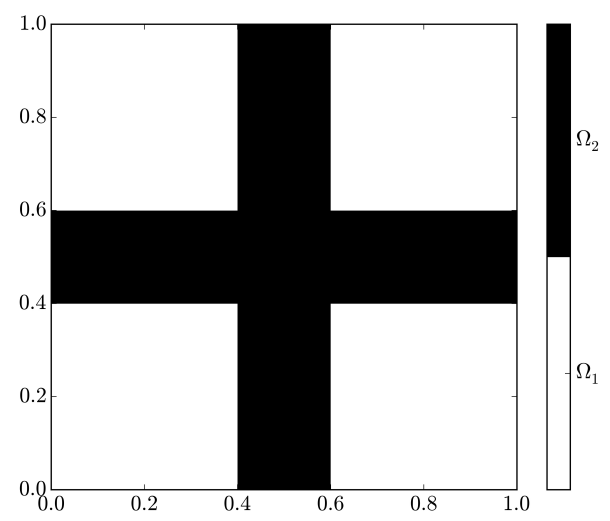

Figure 6: A schematic diagram showing the subdomains $\Omega_{1}$ and $\Omega_{2}$ employed in Example 2 .

The geometry considered in the example is defined as $\Omega_{\ell}=(0,1)^{2}, \Omega_{2}=$ $(0.4,0.6) \times(0,1) \cup(0,1) \times(0.4,0.6)$, and $\Omega_{1}=\Omega_{\ell} \backslash \Omega_{2}$, as shown in Figure 6 . Figure 7 shows representative FE approximations for this example obtained by employing a structured triangular mesh with $161 \times 161$ nodes and polynomial degree $\boldsymbol{k}=(2,2,1)$. In Figures 7(a) and 7(c) we observe complex features of the solution in the region surrounding the points $(0.4,0.4),(0.4,0.6),(0.6,0.4)$, and $(0.6,0.6)$ corresponding to the internal corners of $\Omega_{2}$. These features indicate that there may be a lack of regularity in the underlying continuous solution and, as such, we would not expect to observe the same rates of convergence as attained in Example 1, for which the interface $\Gamma$ was smooth.

For the data employed in this example we do not know an analytical solution, a priori; however, given the interface conditions and periodic boundary conditions we expect that the continuous solution $\boldsymbol{\Phi}$ would satisfy

$$
\left\langle(\boldsymbol{\Phi})_{1}\right\rangle_{1}=0, \quad\left\langle(\boldsymbol{\Phi})_{1}\right\rangle_{2}=0, \quad\left\langle(\boldsymbol{\Phi})_{2}\right\rangle_{1}=0, \quad \text { and } \quad\left\langle(\boldsymbol{\Phi})_{2}\right\rangle_{2} \neq 0
$$

As such, we may investigate the convergence of these quantities numerically. Figure 8 demonstrates the rates of convergence obtained for selected properties of the numerical approximation with respect to the mesh granularity, for a range of polynomial degrees. In Figures $8(\mathrm{a}), 8(\mathrm{c})$, and $8(\mathrm{~d})$ we observe greatly 
reduced rates of convergence compared with those observed in Figure 5 for Example 1. We suggest that a probable cause for this is an underlying lack of regularity in the continuous solution and note that if this is the case, other (non-adaptive) methods would suffer similarly from low rates of convergence. Potentially, application of an $h p$-adaptive method could overcome these issues. Moreover, an analysis of the regularity of the underlying continuous solution would provide further understanding; however, this is beyond the scope of the current work. Figure 8(b) demonstrates the convergence of $\left\langle(\Phi)_{2}\right\rangle_{2}$ to some non-zero quantity at a similar rate, as expected.

\subsection{Macroscale}

In this section we present a representative numerical example of the macroscale model based on the underlying tumour model presented in [18, §5.1]. Prior to this, we note that in order to verify the implementation of the macroscale model discretization presented in sections 3.2.1 and 3.2.2 we confirmed the following for synthetic boundary and forcing data.

- Optimal rates of convergence to a known solution are attained with respect to mesh granularity and polynomial degree for the flow equations.

- Optimal rates of convergence to a known solution are attained for a stationary problem with both zero and non-zero reaction terms.

- A front is propagated by the transport equations through the domain with the correct speed (to within some given tolerance).

- For the evolution of the cell volume fractions, their sum remains one (to within some given tolerance) at all time points.

\subsubsection{Parameterization via Solution of Microscale Problem}

We consider now the microscale problem employed in the parameterization of the macroscale numerical experiments presented in section 4.2. In order for us to obtain meaningful average permeabilities in both subdomains, it is insufficient to consider two-dimensional geometries. As such, in the companion article [18], 


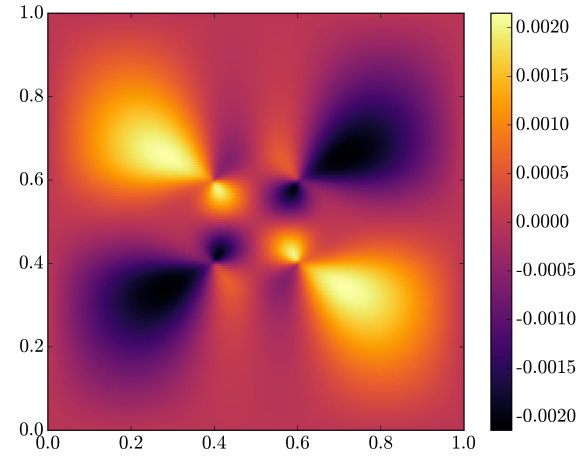

(a) $\left(\boldsymbol{\Phi}_{h, \boldsymbol{k}}\right)_{1}$.

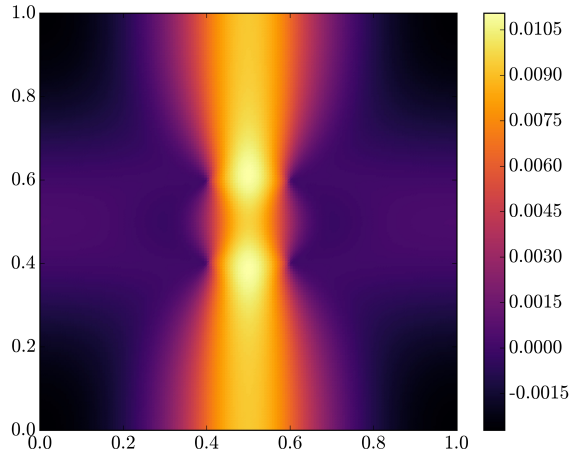

(b) $\left(\boldsymbol{\Phi}_{h, \boldsymbol{k}}\right)_{2}$.

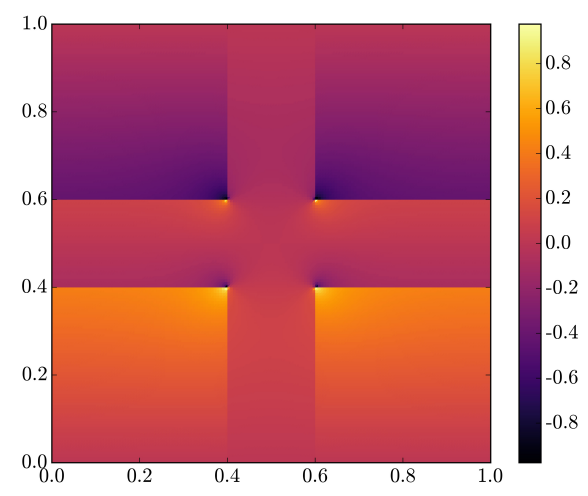

(c) $\phi_{h, k}$.

Figure 7: The FE approximation $\left(\boldsymbol{\Phi}_{h, \boldsymbol{k}}, \phi_{h, \boldsymbol{k}}\right)$ for Example 2, obtained by employing a structured triangular mesh of $161 \times 161$ nodes with polynomial degree $\boldsymbol{k}=(2,2,1)$. 

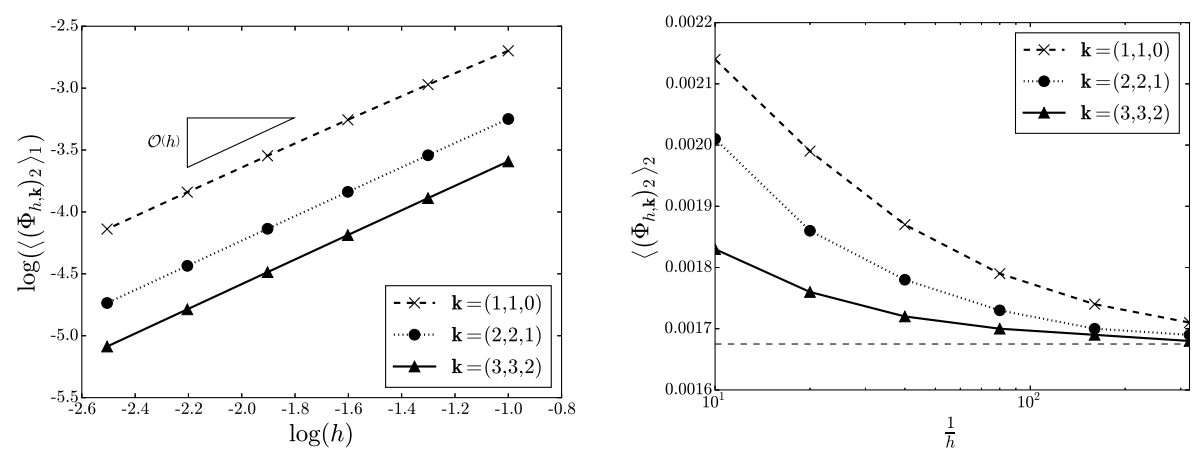

(a) Convergence of the error in (b) Convergence of $\left\langle\left(\boldsymbol{\Phi}_{h, \boldsymbol{k}}\right)_{2}\right\rangle_{2}$. $\left\langle\left(\boldsymbol{\Phi}_{h, \boldsymbol{k}}\right)_{2}\right\rangle_{1}$.
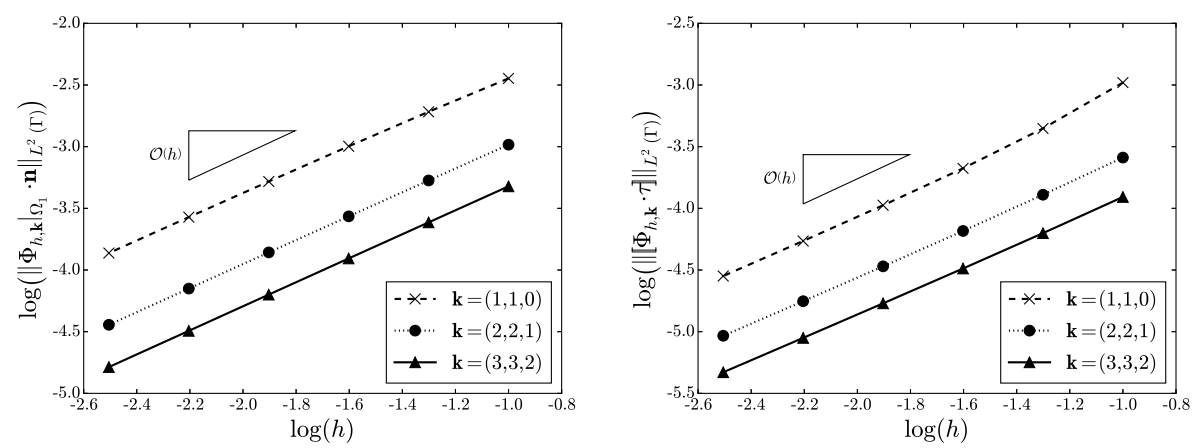

(c) Convergence of the $L^{2}(\Gamma)$ error of the (d) Convergence of the $L^{2}(\Gamma)$ error of the no-penetration condition across $\Gamma$, in $\Omega_{1}$. slip condition on $\Gamma$.

Figure 8: Plots demonstrating the convergence of a selection of quantities obtained from the FE approximation of $(\boldsymbol{\Phi}, \phi)$ for Example 2. 
we consider a three-dimensional geometry and restrict the resulting permeability for employment within two-dimensional macroscale problems. Correspondingly, we define $\Omega_{\ell}=(0,1)^{3}$, and further define $\mathcal{A}_{1}=(0.4,0.6) \times(0.4,0.6) \times(0,1)$, $\mathcal{A}_{2}=(0.4,0.6) \times(0,1) \times(0.4,0.6)$, and $\mathcal{A}_{3}=(0,1) \times(0.4,0.6) \times(0.4,0.6)$. We subsequently define $\Omega_{2}=\bigcup_{i=1}^{3} \mathcal{A}_{i}$ and $\Omega_{1}=\Omega_{\ell} \backslash \bar{\Omega}_{2}$. We then compute an approximate permeability $\boldsymbol{K}_{h, \boldsymbol{k}}$, employing the discretization described in section 3.1, and then restrict the resulting averaged permeability tensor to two dimensions, yielding

$$
\langle\boldsymbol{K}\rangle_{1}=\left(\begin{array}{rr}
2.69 \times 10^{-2} & 2.21 \times 10^{-9} \\
-2.21 \times 10^{-9} & 2.69 \times 10^{-2}
\end{array}\right)
$$

and

$$
\langle\boldsymbol{K}\rangle_{2}=\left(\begin{array}{cc}
4.97 \times 10^{-5} & 3.75 \times 10^{-14} \\
-3.75 \times 10^{-14} & 4.97 \times 10^{-5}
\end{array}\right) .
$$

Given the relatively small size of the off-diagonal entries we consider these as zero in the parameterization of the macroscale model. Further details regarding this computation may be found in [18]; however, as these details are not of direct relevance to this study we do not discuss them further here.

\subsubsection{Computational Results}

In this section we present a representative numerical example of the macroscale model, based on the model described in [18, §5.1], and obtained employing the discretization described in section 3.2. A description of the reaction terms $\mathcal{R}_{\theta, i}, \mathcal{R}_{c}$, and $\mathcal{R}_{n}$, the source terms $g_{\theta, i}$ and the forcing term $f$ may be found in appendix A, together with the values of model parameters employed in the computations presented here, though we refer the reader to the reference for a full discussion regarding the biological motivation for terms of this form.

We consider three phases in the mixture corresponding to normal cells, tumour cells, and extra-cellular matrix (ECM), denoted by $i=1,2,3$, respectively. We define the macroscale geometry employed in the simulations as $\Omega_{L}=\left\{\boldsymbol{X}: X_{1}^{2}+X_{2}^{2} \leqslant 0.5^{2}\right\}$. This is discretized into a shape-regular conforming triangulation, $\mathcal{T}_{h}$, shown in Figure 9 . Note that there is a discrepancy be- 
tween the triangulation and the true domain, but we assume this is sufficiently small to have no qualitative effect on the results, and minimal quantitative effect.

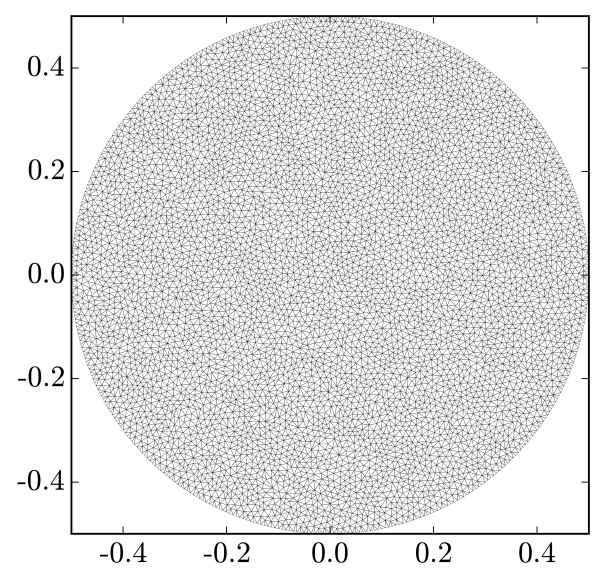

Figure 9: The triangulation, $\mathcal{T}_{h}$, employed in the macroscale flow and transport computations.

We specify the boundary conditions as

$$
\begin{array}{ll}
p=0 & \forall \boldsymbol{X} \in \partial \Omega_{L}, \\
\theta_{1}=\Theta & \forall \boldsymbol{X} \in \Lambda^{-}(\tilde{\boldsymbol{u}}), \\
\theta_{2}=0 & \forall \boldsymbol{X} \in \Lambda^{-}(\tilde{\boldsymbol{u}}), \\
\theta_{3}=1-\Theta & \forall \boldsymbol{X} \in \Lambda^{-}(\tilde{\boldsymbol{u}}), \\
c=c_{\partial}(t) & \forall \boldsymbol{X} \in \Lambda^{-}(\boldsymbol{u}), \\
n=1 & \forall \boldsymbol{X} \in \Lambda^{-}(\boldsymbol{u}),
\end{array}
$$

where $\Theta$ denotes the volume fraction of healthy cells in the tissue surrounding the tumour which we set to 0.75 , and $c_{\partial}(t)$ is a time-varying concentration corresponding to multiple rounds of treatment defined by

$$
c_{\partial}(t)=2\left(\exp \left(-2(t-4)^{2}\right)+\exp \left(-2(t-8)^{2}\right)\right)
$$

We further specify the initial conditions for the volume fractions of the mixture 
components as

$$
\left.\begin{array}{rl}
\theta_{1}(\boldsymbol{X}, 0) & =\Theta-\theta_{2}(\boldsymbol{X}, 0) \\
\theta_{2}(\boldsymbol{X}, 0) & =\Theta\left(\exp \left(-b \boldsymbol{X}^{\top} \boldsymbol{X}\right)-\exp \left(-b a^{2}\right)\right), \\
\theta_{3}(\boldsymbol{X}, 0) & =1-\Theta
\end{array}\right\}
$$

for $\boldsymbol{X} \in\left\{\left(X_{1}, X_{2}\right): X_{1}^{2}+X_{2}^{2} \leqslant a^{2}\right\}$, and

$$
\left.\begin{array}{l}
\theta_{1}(\boldsymbol{X}, 0)=\Theta \\
\theta_{2}(\boldsymbol{X}, 0)=0 \\
\theta_{3}(\boldsymbol{X}, 0)=1-\Theta
\end{array}\right\}
$$

otherwise, where $a=0.45$ and $b=20$, and the initial condition for drug and nutrient concentrations are given by

$$
c(\boldsymbol{X}, 0)=0 \quad \text { and } \quad n(\boldsymbol{X}, 0)=1 .
$$

We specify the rate of phase change on the surface $\Gamma$ to be given by

$$
G=k_{G}^{3}\left(\theta_{3}-\theta_{E}\right)+k_{G}^{2} \mathcal{H}\left(\theta_{2}-\theta_{\mathrm{th}}\right)\left(\theta_{2}-\theta_{\mathrm{th}}\right),
$$

where $\mathcal{H}(\cdot)$ denotes the Heaviside function. The constitutive form of $G$ is an extension of that presented in [18]. The first term represents the process described in the reference, and the second is included as a means of incorporating crowding effects. Finally, we specify the time-step size $\Delta t=0.0002$ and polynomial degree $k=0$ are used for the transport equations throughout the proceeding computational results. Table 1 contains the values employed in the computation of the numerical results.

Figures 10 and 11 show the volume fraction of each of the components of the mixture, and the concentration of drug and nutrient, respectively, for a range of times up to $t=14$. In Figure 10(a) and 10(b), we observe an initial growth of the tumour, which results in a reduction of healthy tissue and ECM. Further, in 11(b) we observe increased consumption of nutrient in the region with high volume fraction of tumour cells compared to the remainder of the domain. In Figures 10(c)-10(e) we observe cell death resulting from the transport of drug through the domain, as observed in Figures 11(a), 11(c), 11(e), 
and 11(g). Throughout Figure 11, we observe increase (decrease) in drug and nutrient concentration in the regions of the subdomain in which compression (rarefaction) occurs as a result of volume changes, associated cell growth and death, and phase transition on the interface $\Gamma$. We remark further that these results are qualitatively consistent with the numerical results presented in [18] and other studies of tumour growth (e.g. [24]).

\section{Conclusions}

In this article we have set out discretizations for the two-scale model of avascular tumour growth and response, and solute transport obtained via multiscale analysis presented in our companion article [18]. The dG FE discretization we propose for the microscale tensor Stokes problem incorporates a non-standard coupling across the interface between the multicomponent mixture and the interstitial fluid. We have been able to demonstrate optimal rates of convergence for sufficiently smooth data. For the macroscale problem, we describe a coupled dG/RT FE discretization for the flow, and dG FE for the solute transport and phase volume fractions, discretized in time employing an explicit Euler method. We have then provided representative numerical experiments in order to demonstrate that these methods can be applied successfully to models of drug transport and tumour response. We note, however, that these techniques should be equally successful in other application domains which require the coupling of flows across a singular interface.

There are several natural extensions to the work in this article. It is important to consider the convergence of the $\mathrm{dG} \mathrm{FE}$ method proposed in section 3.1. However, this analysis will undoubtedly raise questions regarding the wellposedness of the underlying PDE problem, as well as the need for estimates of the regularity of the continuous solution. Finally, we highlight that if it is possible to remove the strong drag assumption in [18], then the methods presented here will need to be supplemented in order to approximate solutions of the resulting microscale system of parametric PDEs, that will likely be posed 
in $3+N_{\theta}$ dimensions.

\section{Acknowledgements}

The first and second authors wish to acknowledge the support of EPSRC grant number EP/K039342/1.

\section{A. Tumour Growth Model}

In this appendix we describe the form of the reaction, forcing, and source terms in the macroscale model introduced in section 2.3 and present a table containing the parameter values employed in the macroscale numerical experiments of section 4.2. Here, we forgo extensive discussion regarding the biological motivation for the form of these terms, and refer the reader to $[18,27]$. We recall from section 4.2 that we consider three phases in the mixture, and proceed by first defining the reaction terms $\mathcal{R}_{\theta, i}$, and source terms $g_{\theta, i}$, associated with the volume fractions of the components of the mixture introduced in (15), by

$$
\begin{aligned}
& \mathcal{R}_{\theta, 1}=-\frac{\rho_{1,3}}{\rho_{1,1}} k_{m, 1} \theta_{3}\left(\frac{n}{n_{P}+n}\right)+k_{d, 1}\left(\frac{n_{1}+n}{n_{2}+n}\right)+k_{c, 1}\left(\frac{c}{c_{P}+c}\right)+\mathcal{S}, \\
& g_{\theta, 1}= 0, \\
& \mathcal{R}_{\theta, 2}=-\frac{\rho_{1,3}}{\rho_{1,2}} k_{m, 2} \theta_{3}\left(\frac{n}{n_{P}+n}\right)+k_{d, 2}\left(\frac{n_{1}+n}{n_{2}+n}\right)+k_{c, 2}\left(\frac{c}{c_{P}+c}\right)+\mathcal{S}, \\
& g_{\theta, 2}= 0, \\
& \mathcal{R}_{\theta, 3}=\left(k_{m, 1} \theta_{1}+k_{m, 2} \theta_{2}\right)\left(\frac{n}{n_{P}+n}\right)+\mathcal{S}, \\
& g_{\theta, 3}=\left(\frac{\rho_{1,1}}{\rho_{1,3}} k_{d, 1} \theta_{1}+\frac{\rho_{1,2}}{\rho_{1,3}} k_{d, 2} \theta_{2}\right)\left(\frac{n_{1}+n}{n_{2}+n}\right)+ \\
& \quad\left(\frac{\rho_{1,1}}{\rho_{1,3}} k_{c, 1} \theta_{1}+\frac{\rho_{1,2}}{\rho_{1,3}} k_{c, 2} \theta_{2}\right)\left(\frac{c}{c_{P}+c}\right),
\end{aligned}
$$

where $\mathcal{S}$ is defined as

$$
\begin{aligned}
\mathcal{S}= & \left(\left(\frac{\rho_{1,3}}{\rho_{1,1}}-1\right) k_{m, 1} \theta_{1}+\left(\frac{\rho_{1,3}}{\rho_{1,2}}-1\right) k_{m, 2} \theta_{2}\right) \theta_{3}\left(\frac{n}{n_{P}+n}\right)+ \\
& \left(\left(\frac{\rho_{1,1}}{\rho_{1,3}}-1\right) k_{d, 1} \theta_{1}+\left(\frac{\rho_{1,2}}{\rho_{1,3}}-1\right) k_{d, 2} \theta_{2}\right)\left(\frac{n_{1}+n}{n_{2}+n}\right)+ \\
& \left(\left(\frac{\rho_{1,1}}{\rho_{1,3}}-1\right) k_{c, 1} \theta_{1}+\left(\frac{\rho_{1,2}}{\rho_{1,3}}-1\right) k_{c, 2} \theta_{2}\right)\left(\frac{c}{c_{P}+c}\right) .
\end{aligned}
$$


The forcing term introduced in the definition of the flow system (13) is given by

$$
f=\mathcal{G}+\mathcal{S}
$$

where, for a specified interfacial phase transition $G$ rate, $\mathcal{G}$ denotes

$$
\mathcal{G}=\left(\frac{1}{\rho_{1}}-1\right)\langle G\rangle_{\Gamma} .
$$

Finally, the reaction terms for the drug and nutrient introduced in (19) and (22), respectively, are given by

$$
\mathcal{R}_{c}=\phi \gamma_{1, c}+\xi_{c}+\mathcal{G}+\mathcal{S}
$$

and

$$
\mathcal{R}_{n}=\phi \gamma_{1, c}+\mathcal{G}+\mathcal{S},
$$

where

$$
\hat{\gamma}_{1, c}\left(\theta_{1}+\theta_{2}\right)
$$

and

$$
\gamma_{1, n}=k_{\gamma, 1} \theta_{1}+k_{\gamma, 2} \theta_{2}+\left(k_{\gamma, 1}^{m} \theta_{1}+k_{\gamma, 2}^{m} \theta_{2}\right) \theta_{3}\left(\frac{n}{n_{P}+1}\right) .
$$

Table 1 contains the values employed in the computation of the numerical results presented in section 4.2, as well as a short description as to the biological interpretation of each parameter. Though, as discussed previously, we refer the reader to [18] and the references therein, for a full discussion surrounding the biological interpretation of the parameters.

[1] T. Alarcón, H. M. Byrne, and P. K. Maini. A cellular automaton model for tumour growth in inhomogeneous environment. Journal of Theoretical Biology, 225:257-274, 2003.

[2] T. Alarcón, H. M. Byrne, and P. K. Maini. Towards whole-organ modelling of tumour growth. Progress in Biophysics $\mathscr{E}$ Molecular Biology, 85:451-472, 2004 .

[3] T. Alarcón, H. M. Byrne, and P. K. Maini. A multiple scale model for tumor growth. Multiscale Modeling \& Simulation, 3(2):440-475, 2005. 


\begin{tabular}{ccl}
\hline Parameter & Value & Description \\
\hline$k_{m, 1}$ & 1.0 & Healthy cell birth rate \\
$k_{m, 2}$ & 3.0 & Tumour cell birth rate \\
$k_{d, 1}$ & 0.28 & Healthy cell death rate, $n$ regulated \\
$k_{d, 2}$ & 0.5 & Tumour cell death rate, $n$ regulated \\
$k_{c, 1}$ & 0.25 & Healthy cell death rate, $c$ regulated \\
$k_{c, 2}$ & 1.5 & Tumour cell death rate, $c$ regulated \\
$k_{\gamma, 1}$ & 0.1 & Nutrient consumption rate (healthy cell baseline) \\
$k_{\gamma, 2}$ & 0.1 & Nutrient consumption rate (tumour cell baseline) \\
$k_{\gamma, 1}^{m}$ & 0.5 & Nutrient consumption rate (healthy cell birth) \\
$k_{\gamma, 2}^{m}$ & $k_{\gamma, 1} \times k_{m, 2}$ & Nutrient consumption rate (tumour cell birth) \\
$\tilde{\gamma}_{1, c}$ & 0.075 & Drug consumption rate \\
$\xi_{c}$ & 0.05 & Drug decay rate \\
$n_{P}$ & 0.25 & Cell birth rate dependence on nutrient \\
$n_{1}, n_{2}$ & $1.0,0.4$ & Cell death rate dependence on nutrient \\
$c_{P}$ & 0.25 & Cell death rate dependence on drug \\
$\phi$ & 0.896 & Porosity \\
$\rho_{1,1}$ & 0.4 & Density of healthy cells \\
$\rho_{1,2}$ & 0.4 & Density of tumour cells \\
$\rho_{1,3}$ & 0.8 & Density of ECM \\
$k_{G}^{3}$ & 3.84 & Constant in assumption for $G$ \\
$\theta_{E}$ & 0.25 & Equilibrium value for $\theta_{3}$ \\
$k_{G}^{2}$ & 4.0 & Constant in assumption for $G$ \\
$\theta_{\mathrm{th}}$ & 0.78 & Threshold value of $\theta_{2}$ \\
\hline & & \\
& 0.4 & \\
\hline
\end{tabular}

Table 1: Parameter values employed in the numerical experiment 
[4] T. Alarcón, M. R. Owen, H. M. Byrne, and P. K. Maini. Multiscale modelling of tumour growth and therapy: The influence of vessel normalisation on chemotherapy. Computational and Mathematical Methods in Medicine, $7(2-3): 85-119,2006$.

[5] P. R. Amestoy, I. S. Duff, J.-Y. L'Excellent, and J. Koster. A fully asynchronous multifrontal solver using distributed dynamic scheduling. SIAM J. Matrix Analysis and Applications, 23(1):15-41, 2001.

[6] P. R. Amestoy, A. Guermouche, J.-Y. L'Excellent, and S. Pralet. Hybrid scheduling for the parallel solution of linear systems. Parallel Computing, 32(2):136-156, 2006.

[7] P. Antonietti, S. Giani, E. Hall, P. Houston, and R. Krahl. AptoFEM documentation. http://www.aptofem.com, June 2015.

[8] P. F. Antonietti, A. Cangiani, J. Collis, Z. Dong, E. H. Georgoulis, S. Giani, and P. Houston. Review of Discontinuous Galerkin finite element methods for partial differential equations on complicated domains. Accepted for publication, 2015.

[9] R. P. Araujo and D. L. S. McElwain. A history of the study of solid tumour growth: The contribution of mathematical modelling. Bulletin of Mathematical Biology, 66:1039-1091, 2004.

[10] D. Bresch and J. Koko. Operator-splitting and Lagrange multiplier domain decomposition methods for numerical simulation of two coupled NavierStokes fluids. Internationa Journal of Applied Mathematics and Computer Science, 16(4):419-429, 2006.

[11] C. J. W. Breward, H. M. Byrne, and C. E. Lewis. A multiphase model describing vascular tumour growth. Bulletin of mathematical biology, 1:128,2004 .

[12] F. Brezzi. Numerical Analysis, chapter Interacting with the Subgrid World, pages $69-82$. CRC, 2000. 
[13] F. Brezzi, J. Douglas, R. Duran, and M. Fortin. Mixed finite element methods for second order elliptic problems in three variables. Numer. Math., $51: 237-250,1987$.

[14] F. Brezzi, J. Douglas, M. Fortin, and L. D. Marini. Efficient rectangular mixed finite elements in two and three space variables. Math. Model. Numer. Anal., 21:581 - 604, 1987.

[15] F. Brezzi, J. Douglas, and L. D. Marini. Recent results on mixed finite element methods for second order elliptic problems. In Balakrishanan, Dorodnitsyn, and Lions, editors, Vistas in Applied Math, Numerical Analysis, Atmospheric Sciences, Immunology. Optimization Software Publications, New York, 1986.

[16] F. Brezzi and M. Fortin. Mixed and Hybrid Finite Element Methods. Springer-Verlag, New York, 1991.

[17] B. Cockburn, G. E. Karniadakis, and C.-W. Shu. The development of discontinuous Galerkin methods. In B. Cockburn, G. E. Karniadakis, and C.-W. Shu, editors, Discontinuous Galerkin Finite Element Methods, volume 11 of Lect. Notes Comput. Sci. Eng., pages 3-50. Springer-Verlag, Berlin, 2000.

[18] J. Collis, M. E. Hubbard, and R. D. O'Dea. A multiscale analysis of drug transport and response for a multiphase tumour model. Submitted.

[19] Y. Davit, C. G. Bell, H. M. Byrne, L. A. C. Chapman, L. S. Kimpton, G. E. Lang, K. H. L. Leonard, J. M. Oliver, N. C. Pearson, R. J. Shipley, S. L. Waters, J. P. Whiteley, B. D. Wood, and M. Quintard. Homogenization via formal multiscale asymptotics and volume averaging: How do the two techniques compare? Advances in Water Resources, 62:178-206, 2013.

[20] M. Discacciati, P. Gervasio, A. Giacomini, and A. Quarteroni. The interface control domain decomposition method for stokes-darcy coupling. SIAM J. Numer. Anal., 54(2):1039-1068, 2016. 
[21] M. Discacciati, P. Gervasio, and A. Quarteroni. Interface control domain decomposition methods for heterogeneous problems. Int. J. Numer. Meth. Fluids, 76(8):471-496, 2014.

[22] Y. Efendiev and T. Y. Hou. Multiscale Finite Element Methods: Theory and Applications. Springer, New York, 2009.

[23] C. Farmer. Upscaling: A review. Int. J. Numer. Meth. Fluids, 40:63 - 78, 2002.

[24] S. J. Franks and J. R. King. Interactions between a uniformly proliferating tumour and its surroundings. Mathematical Medicine and Biology, 20:4789, 2003.

[25] H. B. Frieboes, J. S. Lowengrub, S. M. Wise, X. Zheng, P. Macklin, E. L. Bearer, and V. Cristini. Computer simulation of glioma growth and morphology. Neuroimage, 37:S58 - S70, 2007.

[26] P. Houston and E. Süli. hp-adaptive discontinuous Galerkin finite element methods for first-order hyperbolic problems. SIAM J. Sci. Comput., 23(4):1226-1252, 2001.

[27] M. E. Hubbard and H. M. Byrne. Multiphase modelling of vascular tumour growth in two spatial dimensions. Journal of Theoretical Biology, 316:7089, 2013.

[28] J. B. Keller. Darcy's law for flow in porous media and the two-scale method. In R. L. Sternberg, A. J. Kalinowski, and J. S. Papadakis, editors, Nonlinear PDE in Engineering and Applied Sciences. Marcel Dekker, 1980.

[29] P. Macklin, S. McDougall, A. R. A. Anderson, M. A. J. Chaplain, V. Cristini, and J. Lowengrub. Multiscale modelling and nonlinear simulation of vascular tumour growth. Journal of Mathematical Biology, 58(45):765-798, 2009.

[30] J. C. Nedelec. Mixed finite elments in $\mathbb{R}^{3}$. Numer. Math., 35:315-341, 1980. 
[31] R. D. O’Dea, M. R. Nelson, A. J. El Haj, S. L. Waters, and H. M. Byrne. A multiscale analysis of nutrient transport and biological tissue growth in vitro. Mathematical Medicine and Biology, 2014.

[32] R. D. O'Dea, S. L. Waters, and H. M. Byrne. A two-fluid model for tissue growth within a dynamic flow environment. European Journal of Applied Mathematics, 19:607-634, 2008.

[33] M. R. Owen, T. Alarcón, P. K. Maini, and H. M. Byrne. Angiogenesis and vascular remodelling in normal and cancerous tissues. Journal of Mathematical Biology, 58(4-5):689-721, 2009.

[34] M. R. Owen, I. J. Stamper, M. Muthana, G. W. Dobson, C. E. Lewis, and H. M. Byrne. Mathematical modeling predicts synergistic antitumor effects of combining a macrophage-based, hypoxia-targeted gene therapy with chemotherapy. Cancer Research, 71(8):2826-2837, 2011.

[35] H. Perfahl, H. M. Byrne, T. Chen, V. Estrella, T. Alarcón, A. Lapin, R. A. Gatenby, R. J. Gillies, M. C. Lloyd, P. K. Maini, M. Reuss, and M. R. Owen. Multiscale modelling of vascular tumour growth in 3d: the roles of domain size and boundary conditions. PloS one, 6(4):e14790, 2011.

[36] G. G. Powathil, M. Swat, and M. A. J. Chaplain. Systems oncology: Towards patient-specific treatment regimes informed by multiscale mathematical modelling. Seminars in cancer biology, 30:13-20, 2015.

[37] M. Ptashnyk and T. Roose. Derivation of a macroscopic model for transport of strongly sorbed solutes in the soil using homogenization theory. SIAM Journal on Applied Mathematics, 70(7):2097-2118, 2010.

[38] P. A. Raviart and J. M. Thomas. A mixed finite element method for second order elliptic problems. In I. Galligani and E. Magenes, editors, Mathematical Aspects of the Finite Element Method, Lectures Notes in Math. 606. Springer-Verlag, New York, 1977. 
[39] J. Rubinstein and S. Torquato. Flow in random porous media: mathematical formulation, variational principles, and rigorous bounds. J. Fluid Mechanics, 206:25-46, 1989.

[40] J. R. Shewchuk. Triangle: Engineering a 2D Quality Mesh Generator and Delaunay Triangulator. In Ming C. Lin and Dinesh Manocha, editors, Applied Computational Geometry: Towards Geometric Engineering, volume 1148 of Lecture Notes in Computer Science, pages 203-222. SpringerVerlag, 1996. From the First ACM Workshop on Applied Computational Geometry.

[41] R. J. Shipley. Multiscale modelling of fluid and drug transport in vascular tumours. PhD thesis, University of Oxford, 2008.

[42] R. J. Shipley and S. J. Chapman. Multiscale modelling of fluid and drug transport in vascular tumours. Bull. Math. Biol., 72(6):1464-1491, 2010.

[43] R. J. Shipley, S. J. Chapman, and R. Jawad. Multiscale modeling of fluid transport in tumors. Bull. Math. Biol., 70(8):2334-2357, 2010.

[44] A. Toselli. hp-finite element discontinuous Galerkin approximations for the Stokes problem. M3AS, 12:1565-1616, 2002. 

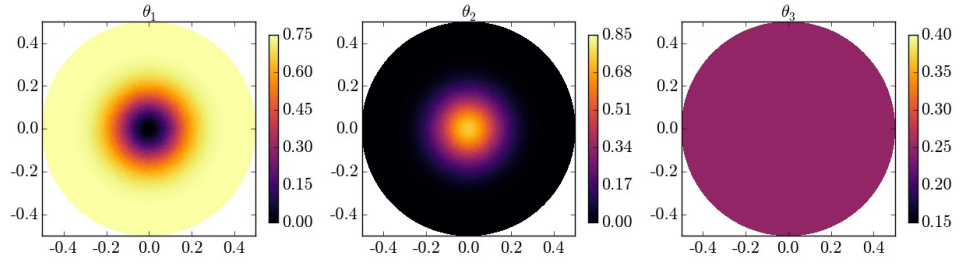

(a) $t=0$.
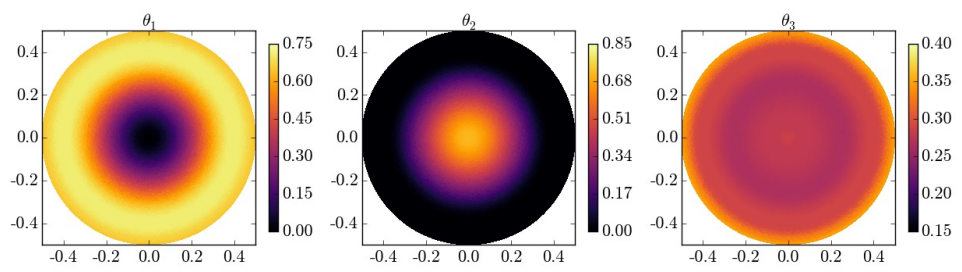

(b) $t=5$.
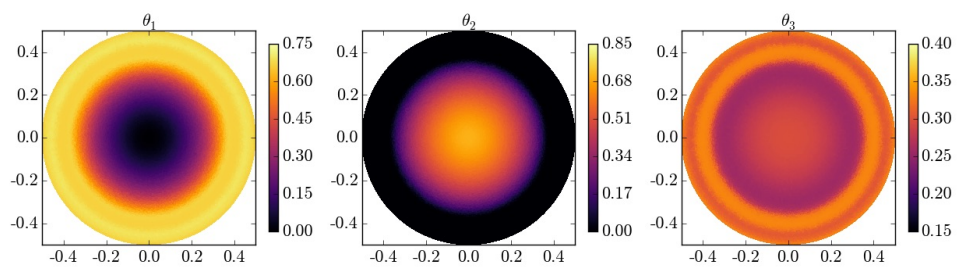

(c) $t=8$.
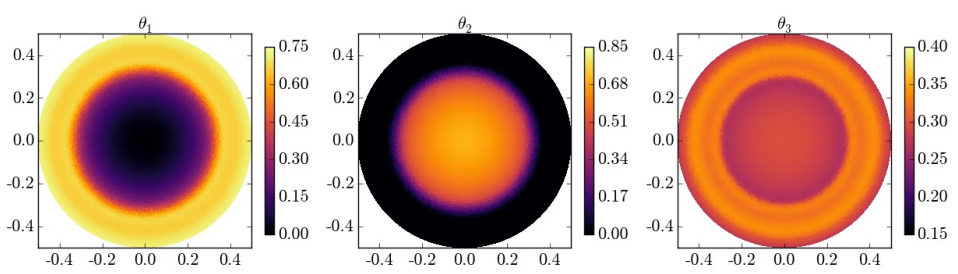

(d) $t=11$
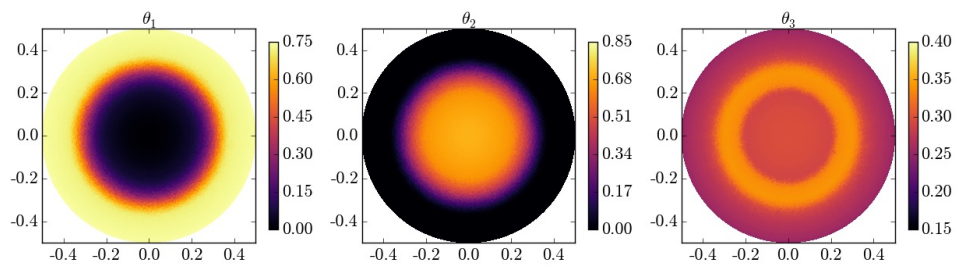

(e) $t=14$.

Figure 10: Numerical approximation of the normal cell $\left(\theta_{1}\right)$, tumour cell $\left(\theta_{2}\right)$, and ECM $\left(\theta_{3}\right)$ volume fractions obtained at $t=0,5,8,11$, and 14 . 


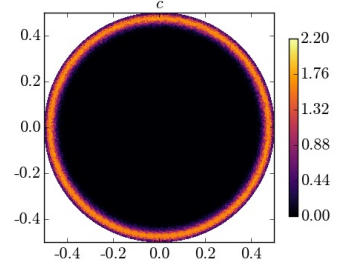

(a) $t=5$.

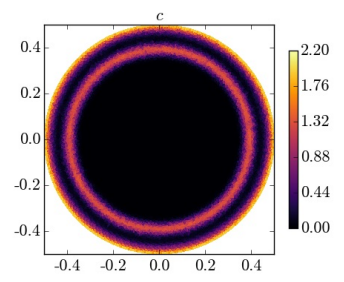

(c) $t=8$.

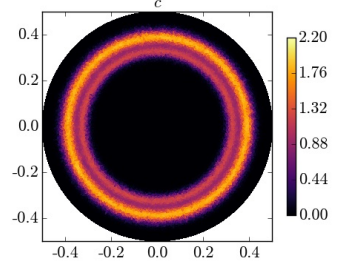

(e) $t=11$.

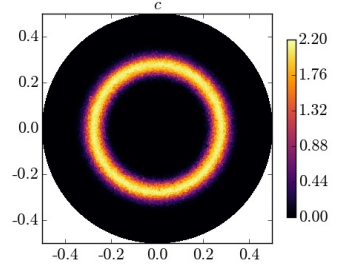

(g) $t=14$.

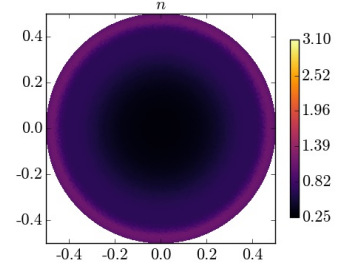

(b) $t=5$.

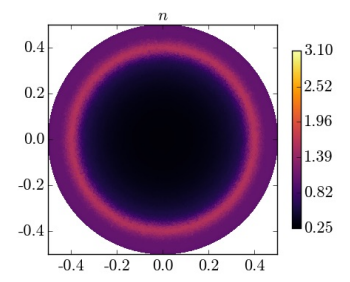

(d) $t=8$.

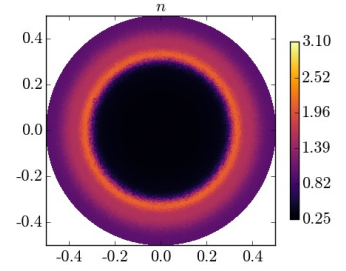

(f) $t=11$.

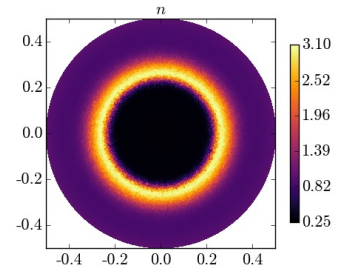

(h) $t=14$.

Figure 11: Numerical approximation of the drug $(c)$ and nutrient $(n)$ concentrations obtained at $t=5,8,11$, and 14 . 